\title{
Strong Activity Rules for Iterative Combinatorial Auctions
}

\section{Citation}

Harsha, Pavithra, Cynthia Barnhart, David C. Parkes, and Haoqi Zhang. 2010. Strong activity

rules for iterative combinatorial auctions. Computers \& Operations Research 37(7): 1271-1284.

\section{Published Version}

doi:10.1016/j.cor.2009.09.003

\section{Permanent link}

http://nrs.harvard.edu/urn-3:HUL.InstRepos:3967325

\section{Terms of Use}

This article was downloaded from Harvard University's DASH repository, and is made available under the terms and conditions applicable to Open Access Policy Articles, as set forth at http:// nrs.harvard.edu/urn-3:HUL.InstRepos:dash.current.terms-of-use\#OAP

\section{Share Your Story}

The Harvard community has made this article openly available.

Please share how this access benefits you. Submit a story.

Accessibility 


\title{
Strong Activity Rules for Iterative Combinatorial Auctions
}

\author{
Pavithra Harsha \\ Oracle Retail, 25 First St, Cambridge, MA 02141, USA \\ Cynthia Barnhart \\ Massachusetts Institute of Technology, Room 1-206, Civil and Environmental Engineering and Operations \\ Research Center, 77 Massachusetts Avenue, Cambridge MA 02139, USA \\ David C. Parkes ${ }^{1}$, Haoqi Zhang \\ Maxwell Dworkin 229, School of Engineering and Applied Sciences, Harvard University, 33 Oxford Street, \\ Cambridge, MA 02138, USA
}

\begin{abstract}
Activity rules have emerged in recent years as an important aspect of practical auction design. The role of an activity rule in an iterative auction is to suppress strategic behavior by bidders and promote simple, continual, meaningful bidding and thus, price discovery. These rules find application in the design of iterative combinatorial auctions for real world scenarios, for example in spectrum auctions, in airline landing slot auctions, and in procurement auctions. We introduce the notion of strong activity rules, which allow simple, consistent bidding strategies while precluding all behaviors that cannot be rationalized in this way. We design such a rule for auctions with budget-constrained bidders, i.e., bidders with valuations for resources that are greater than their ability to pay. Such bidders are of practical importance in many market environments, and hindered from bidding in a simple and consistent way by the commonly used revealed-preference activity rule, which is too strong in such an environment. We consider issues of complexity, and provide two useful forms of information feedback to guide bidders in meeting strong activity rules. As a special case, we derive a strong activity rule for non budget-constrained bidders. The ultimate choice of activity rule must depend, in part, on beliefs about the types of bidders likely to participate in an auction event because one cannot have a rule that is simultaneously strong for both
\end{abstract}

\footnotetext{
${ }^{1}$ Corresponding author: Email - parkes@eecs.harvard.edu, Fax - +1.617.495.8612
} 
budget-constrained bidders and quasi-linear bidders.

Key words: Combinatorial auctions, Activity rules, Budget-constrained bidders, Bidding, Utility-preference: Theory

\section{Introduction}

Combinatorial auctions provide a means of auctioning several related items, allowing bidders to place bids on packages of items rather than individual items. They are used in scenarios such as truckload transportation, bus routes, industrial procurement, and allocation

of radio spectrum, and have been proposed for the allocation of airport landing slots [1]. Among combinatorial auctions, iterative and especially ascending-price auctions are more widely used than their sealed-bid counterparts, due to the feedback and price discovery that they allow $[2]$.

In high-stakes scenarios, such as auctions for the allocation of wireless spectrum [3] or airport landing slots [4], strategic behavior on the part of bidders can lead to large inefficiency. For example, bidders could underbid in the initial phase of the auction with a view to sniping at the end of the auction, which leads to poor price discovery and inefficient outcomes $[5,6]$. This necessitates the use of activity rules to constraint the strategy space as much as possible while still allowing for feedback and price discovery.

The importance of activity rules in suppressing insincere bidding and eliminating them in iterative auctions has been emphasized in practical auction design $[6,7,5]$. Activity rules help in increasing the pace of an auction and increasing the information available to the bidders during an auction. When coupled with careful design of pricing rules, activity rules also help in achieving the efficient outcome with good revenue properties $[8,9,10,11,12]$.

The importance of activity rules has emerged, in part, by the recognition that welldesigned iterative auctions should promote simple "demand-revealing processes," whereby bidders simply demand the items that maximize their utility at the current prices. The idea is to promote simple and consistent bidding, or straightforward bidding, in which there exists a posteriori some (possibly untruthful) utility function that explains the response of bidders in every round. 
We introduce the notion of strong activity rules, which admit straightforward bidding strategies while precluding all strategies that are not consistent with some straightforward strategy. Strong activity rules do not, in any way, preclude the price discovery and demand discovery benefits associated with iterative auctions. Adopting a strong activity rule does not imply that bidders must either know their utility for different items at the start of the auction, or even bid with the same utility in mind in every round. Rather, a strong rule requires that the bids that they do submit are ultimately consistent with at least one utility function; the set of such utility functions that "rationalize" bidding emerge over time. ${ }^{2}$

One popular activity rule requires that the total quantity demanded by a bidder be monotonically non-increasing as prices increase. However, this is inappropriate for combinatorial auctions because it can preclude straightforward bidding, while on the other hand allow for strategic behavior in which a bidder can bid for a large quantity of low value items and then switch to the items really demanded towards the close of the auction. The revealed preference activity rule (RPAR) has been proposed as an alternative [5] and advocated for many practical scenarios, including for use in the upcoming UK spectrum auction [13] and for landing slot auctions at New York airports [14].

But many current day markets such as the cellular and airline industries involve budgetconstrained bidders $[3,15] .^{3}$ Budget-constrained bidders have valuations for resources that are greater than their ability to pay, for instance due to liquidity or credit problems. ${ }^{4}$ Che and Gale [19] also note that budget constraints can result from a problem of moral hazard; many organizations delegate acquisition decisions to purchasing units, while imposing budgets to

\footnotetext{
${ }^{2}$ For the particular case of iterative Vickrey auctions, a strong activity rule ensures that truthful, straightforward bidding is an ex post Nash equilibrium [10, 11, 12].

${ }^{3}$ Airlines typically carry large amounts of debt and are especially vulnerable to fuel spikes, recession or economic shocks [15]. In recent years, all the US airlines together lost over US $\$ 35$ Billion during the period 2001-2005. Four of the six major U.S airlines declared Chapter 11 bankruptcy and have only recently emerged [16]. In wireless spectrum auctions, it is realistic to assume that all firms participating in these auctions face budget constraints $[17,3]$. Bidders must raise funds before the auction starts, a time-consuming and costly process that arguably leads to budget constraints.

${ }^{4}$ Pitchik [18] explains that capital market imperfections limit buyers' abilities to borrow against future income when investments are large.
} 
constrain their spending. ${ }^{5}$ For budget-constrained bidders, RPAR can actually have the opposite effect to that desired, because bidders that bid straightforwardly may fail to satisfy the rule and must instead behave strategically because of the rule. In fact, we show that RPAR is also problematic because it fails to guarantee straightforward bidding for bidders without budget constraints.

Both these drawbacks of RPAR illuminate why the design of activity rules needs to be revisited. From the definition of strong activity rules, we are able to develop activity rules for a broadly applicable family of ascending price auctions, allowing for a variety of different prices including non-linear (i.e., the price of a bundle need not be the sum total price of the constituent items) and non-anonymous (or personalized) prices. In the auctions that we consider, the auctioneer reports prices to bidders in each round and bidders respond with a demand set that defines a package of items. Our activity rules also extend to auctions in which the bidder reports multiple packages of items, across which she is indifferent, in each round, and in which the prices are not necessarily ascending. Given this, our results can find application to many auctions. ${ }^{6}$

We explain how to provide feedback to guide a bidder in meeting our strong activity rules, both in terms of the commitments that a bidder is (implicitly) making about her budget constraint through her bids, and also to guide a bidder in modifying bids in order to pass the rule. An auction designer might in fact prefer to provide somewhat relaxed rules; e.g., for reasons of the complexity of the rules themselves, to allow for some mistakes for bidders, and to allow for some value interdependency and learning by bidders. For this, we advocate as a design principle that one should start with a strong rule and then relax this rule as necessary. Relaxing away from our strong activity rule will certainly allow for straightforward bidding behavior but will, in addition, permit some other behaviors.

In practice, we observe that one cannot have a rule that is both strong for budget-

\footnotetext{
${ }^{5}$ This is observed even for low-valued goods, such as in the domain of sponsored search, in which advertisers can place caps on the amount they are willing to spend on Internet advertisements over a day.

${ }^{6}$ Possible applications include to the combinatorial clock auction [20], the clinching auction [10, 21], RAD [22], $i$ Bundle and ascending-proxy [9, 8], the clock-proxy auction [5], iBEA [11], AkBA [23], and $d V S V[12]$.
} 
constrained and non budget-constrained bidders, and the choice of rule must depend on the bid-taker's beliefs about the utility functions of participants in the auction.

In experimental simulations, we compare RPAR against our strong activity rule for budget-constrained bidders in the clock-proxy auction [5]. This auction is advocated for practical settings such as the FCC wireless spectrum auctions, and consists of an ascendingprice combinatorial clock auction phase that terminates with a "last-and-final" round in which bidders submit additional bids before the auction transitions to a sealed-bid (proxy) auction phase. Given our focus on issues related to the activity rule, we assume for the purpose of our simulations that bidders try to bid straightforwardly, and adopt optimization techniques to modify these bids as necessary when this behavior is blocked by the RPAR rule. This is what we refer to as maximally straightforward bidding.

Details of our results are provided in Section 5. In summary, the strong activity rule outperforms RPAR with respect to efficiency and revenue by $3.8 \%$ and $9.4 \%$ respectively (on average across the different distributions) at low budgets, with benefits falling off as budgets are increased. For certain distributions, we observe efficiency and revenue improvements as high as $13.2 \%$ and $20.3 \%$ respectively, while for other distributions the improvements were not statistically significant, even at low budgets.

Organization of the paper. The rest of the paper is organized as follows. In Section 2, we describe the notation and give some preliminaries, and define the notion of a strong activity rule. In Section 3, we discuss existing activity rules and RPAR in particular, describe some of their features and illustrate some key properties that they fail to achieve. We develop the strong activity rules in Section 4 and compare them with other rules, providing also a discussion about extensions and modifications. In Section 5, we discuss our experimental simulations and finally conclude in Section 6.

\subsection{Related Work}

Auctions with budget constraints have been discussed in many works [24, 19, 25, 26, 27, 18]. However, none of this literature discusses activity rules, and every paper is restricted to domains with at most two items for sale. The focus is instead on equilibrium behavior. In the context of combinatorial auctions, impossibility results exist for truthful, Pareto optimal 
combinatorial auctions in the presence of budget-constrained bidders [28, 29]. On the other hand, Pareto optimal and revenue optimal, sealed-bid auctions have been designed for special cases [29, 30]. Aggarwal et al. [31] develop a stable, incentive-compatible auction that admits budget-constrained bidders for a generalization of the assignment problem. Ausubel and Milgrom [32] also discuss a generalization of ascending-proxy auctions to allow for budgetconstrained bidders. Both of these latter papers are in the context of sealed bid auctions and do not consider the role of activity rules.

Theoretical models for firms with budget constraints allow for both hard and soft (i.e., flexible) budget constraints[33]. Hard budgets are those that cannot be exceeded while soft budget constraints are those that can be exceeded under certain circumstances. Following Kornai et al. [33], a firm can be modeled as hard budget-constrained if it does not receive outside support to cover its deficit and is obliged to reduce or cease its activity if a deficit persists. This is in contrast with soft budgets, where there are supporting agencies that cover all or part of the deficit. ${ }^{7}$ As the notion of soft budgets can vary a lot, for simplicity, we restrict ourselves to bidders with hard budgets.

The notion of a strong activity rule adopts the idea of rationalizability from microeconomics for our purpose. Rationalizability seeks a utility function that explains the observed demand behavior of a consumer in response to varying prices. Notably, Afriat [34] developed simple conditions for rationalizability for a concave utility function where the utility of a package of goods does not depend on the price as long as the package is affordable. ${ }^{8}$ Whereas agents in Afriat's model are indifferent to the price as long as the total expenditure remains within their budget constraint, in our models bidders always prefer to spend less than more. See also Vohra [35], for a discussion of rationalization in the context of quasi-linear utility functions.

Activity rules have been discussed in a number of places in the auction literature. The

\footnotetext{
${ }^{7}$ Loss making state enterprises bailed out by financial subsidies or other instruments are examples of soft budgets.

${ }^{8}$ Afriat's Theorem characterizes conditions for the specific utility function of the form $\max _{x}\{v(x) \mid p \cdot x \leq B\}$ where $v(x)$ represents the values of package $x$ with linear price vector $p$ and budget $B$.
} 
activity rule used in the FCC auction, due to Milgrom and Wilson [6], is a variation on a simple quantity-monotonicity rule, in which quantities that are bid in the auction are required to weakly monotonically decrease across rounds. Similar rules have become standard in combinatorial auctions, perhaps because of their simplicity. For instance, in the combinatorial clock auction [20], a variation on the aggregate monotonicity rule is adopted. In the context of iterative, two-sided markets, Wilson [7] describes activity rules for an auction for electrical power generation, including a bid withdrawal and a revision rule. Mishra and Parkes [11] study a special class of ascending price auctions with quasi-linear bidders and provide (in our terms) strong activity rules with a simple form. Day [36] has previously considered the role of activity rules in the presence of budget constraints, and provides a rule that extends RPAR but is not strong in our sense, because it still allows for some non straightforward bidding (both with and without budget-constrained bidders.)

\section{Strong Activity Rules}

Let $\mathcal{G}$ denote the set of items in an auction and let $\mathcal{I}$ denote the set of bidders. We assume a private values model, with $v_{i}(S) \geq 0$ denoting the value of bidder $i$ in $\mathcal{I}$ for bundle $S \subseteq \mathcal{G}$. We use package and bundle interchangeably in the paper. We normalize $v_{i}(\emptyset)=0$ and assume that the auctioneer is indifferent across all allocations. We assume free-disposal, i.e., $v_{i}(T) \leq v_{i}(S)$ for all $T \subseteq S$, unless it is specifically mentioned.

Let $p_{i}(S) \geq 0$ be the price the auctioneer sets for a bundle $S$ for a bidder $i$. Prices may depend on the bidder if the prices are non-anonymous in nature. Prices are said to be linear if $p_{i}(S)=\sum_{g \in S} p_{i}(\{g\})$ and nonlinear otherwise (i.e., if $p_{i}(S) \neq p_{i}\left(S_{1}\right)+p_{i}\left(S_{2}\right)$ for some $S=S_{1} \cup S_{2}$ and $\left.S_{1} \cap S_{2}=\emptyset\right)$. We will often drop the subscript $i$ in our notation, because the context of the bidder is generally clear.

Bidders are modeled as utility-maximizing, and with a utility function that belongs to a utility domain $\Theta$. An instance $\theta \in \Theta$ captures the set of all parameters that define the utility function $u(S, p)$ for a bidder on bundle $S$ at prices $p$. We consider two models of utility functions:

Quasi-Linear The utility of a bidder for bundle $S$ at price $p$ is given by $u(S, p)=v(S)-$ 
$p(S)$, for some valuation function $v: 2^{\mathcal{G}} \rightarrow \mathbb{R}_{\geq 0}$. A quasi-linear bidder with valuation function $v$ has type $\theta=v$.

Budget-Constrained We consider a model where a bidder has a hard budget constraint and a quasi-linear utility up to the budget. The utility function then has the form:

$$
u(S, p)= \begin{cases}v(S)-p(S) & \text { if } p(S) \leq B \\ -\infty & \text { otherwise }\end{cases}
$$

where $B$ is the bidder's budget. A budget-constrained bidder with valuation $v$ and budget $B$ has type $\theta=(v, B)$. We call this the budget-constrained utility function.

Remark 2.1. In defining budget-constrained bidders, we choose to consider only a class of bidders in which the budget constraint is uniform across all bundles, and invariant over the course of an auction. In order to extend our methods to handle a budget constraint that varies with prices, one would need to make some additional modeling assumptions about the dependence of such an effect on prices. While a budget constraint that depends on the particular bundle could also be handled, the effect would be to allow for significantly more bid flexibility (and thus too much flexibility for bidders with a more restricted utility type), while also making it more challenging to generate the implicit budget feedback that we can construct for the current model.

An ascending price auction is an auction with a single price path that is non-decreasing such that it ends with an allocation and payment for bidders [37, 2, 11]. We focus in particular on auctions in which the auctioneer specifies prices in each round and each bidder responds with a report about a package of items that maximizes her utility given the current prices. ${ }^{9}$ We generalize this later to allow for auction designs in which bidders must report multiple (indifference) sets of packages. Prices are incremented from round to round by the auctioneer. The auction continues until a termination condition is met.

\footnotetext{
${ }^{9}$ Our work also extends to the case of an open outcry auction with jump bids, wherein bids include a bid price that may be greater than the current ask price. In this case, the activity rule takes the price of a bundle as the maximum of the ask price and the bid price (from the jump bid).
} 
At the end of every round, we associate with each bidder a set of price-bid pairs, $(p, S)$, where $p: 2^{\mathcal{G}} \rightarrow \mathbb{R}$ and $S \subseteq \mathcal{G}$, also known as the history of the bidder. History $h$ of a bidder is the set $h=\left\{\left(p^{1}, S^{1}\right), \ldots,\left(p^{t}, S^{t}\right)\right\}$, where $S^{i}$ is the bid placed by the bidder when the prices are $p^{i}$ and $t$ is the cumulative number of bids placed by the bidder in all the rounds of the auction including the current round. Let $H$ denote the set of histories.

We will not place any restrictions on the types of prices, (linear or non-linear, anonymous or non-anonymous), or on the particular method by which prices are increased across rounds (ascending or non-ascending). Note that this may be more than the number of rounds, for instance in the case in which multiple bundles receive bids in a single round.

An activity rule is a rule to check whether the history of each bidder satisfies some set of constraints. More formally, an activity rule, $A$, is specified by a function $f_{A}: H \rightarrow\{0,1\}$ where history $h \in H$ satisfies the rule if $f_{A}(h)=1$ and violates the activity rule if $f_{A}(h)=0$. If a bidder does not satisfy the activity rule in some round then some penalty is imposed, for instance, the bidder is precluded from bidding further or a default bid is submitted.

At a given price, we say a bidder bids straightforwardly if the bundle(s) she bids on are utility maximizing with respect to some utility function. Such a bidder need not be truthful and may bid straightforwardly with respect to some non-truthful utility function. A truthful bidder is a bidder that is straightforward with respect to her true utility function.

Let $h_{\text {bundles }} \in S B\left(h_{\text {prices }}, \theta\right)$ denote a history $h=\left(h_{\text {bundles }}, h_{\text {prices }}\right)$ for a straightforward bidder that adopts utility function $\theta$, where $h_{\text {prices }}$ and $h_{\text {bundles }}$ refer to the price and bundle components of the history. A bidder is consistent given history $h$ if there exists a utility function $\theta \in \Theta$ such that the observed history $h=\left(h_{\text {bundles }}, h_{\text {prices }}\right)$ satisfies $h_{\text {bundles }} \in S B\left(h_{\text {prices }}, \theta\right)$. Consistency requires that there exists a single utility function that explains the bids of the bidder, under straightforward behavior.

Definition 2.2. Strong Activity Rule: An activity rule $A$ is said to be strong with respect to utility domain $\Theta$ if the following conditions are satisfied:

Necessary: $\forall \theta \in \Theta$ and $\forall h=\left(h_{\text {bundles }}, h_{\text {prices }}\right) \in H$ such that $h_{\text {bundles }} \in S B\left(h_{\text {prices }}, \theta\right)$, then $f_{A}(h)=1$; and

Sufficient: $\forall h=\left(h_{\text {bundles }}, h_{\text {prices }}\right) \in H$ such that $f_{A}(h)=1$, there exists $a \theta \in \Theta$ such 
that $h_{\text {bundles }} \in S B\left(h_{\text {prices }}, \theta\right)$.

The necessary conditions state that any consistent bidder with utility in domain $\Theta$ will satisfy the constraints. In this sense, the rule is not too strong. The sufficiency conditions state that if a bidder satisfies the constraints, then the bidder is consistent with respect to some utility function in domain $\Theta$. In this sense, the rule is not too weak.

A strong activity rule is the best that we can do in the following sense: if a rule satisfies the sufficient property then no behavior that it allows can be precluded without precluded a straightforward bidder for some utility in domain $\Theta$. If the rule then also satisfies the necessary property, then it does not need to be relaxed to admit more behaviors because all desired behaviors are allowed. ${ }^{10}$

\section{Monotonicity and Revealed-Preference Activity Rules}

In this section we demonstrate that the common activity rules of aggregate monotonicity and revealed-preference fail to be strong rules.

\subsection{Aggregate Monotonicity Rule}

One common activity rule requires that bids are quantity-monotonic, i.e. as the price increases the total quantity of items bid by each bidder has to decrease. We refer to this rule as the Monotonicity Rule (MR). Defined in a setting with multiple identical items, it is easy to see that a truthful bidder with quasi-linear utility will satisfy MR. Indeed, in Section 4 we will see that MR is a strong activity rule for multiple-identical items in auctions with linear prices. On the other hand, when coupled with non-linear prices, a straightforward, non budget-constrained bidder need not satisfy MR and thus the rule is too strong. This is illustrated with the following simple example:

Example 3.1. Consider a bidder, with values 12 and 15 for one unit and two units of the good. Suppose the prices for the two different bundles in round 1 are 5 and 9, and in round

\footnotetext{
${ }^{10}$ One can also understand the role of these two requirements by noticing that the null rule that always admits any bidding behavior is necessary, while the rule that never admits any bidding behavior is sufficient.
} 
2 are 8 and 10 respectively. Note that the prices are chosen to allow for volume discounts for the substitutable items. At these prices the bidder, when bidding straightforwardly with respect to her true values, demands one unit in round 1 and two units in round 2. Thus a truthful bidder would violate the Monotonic Rule and so be unable to express her true demand.

This problem with MR is well understood and continues even in a setting with distinct items. The appropriate form of the rule in this setting is the Aggregate Monotonicity Rule $(A M R)$, in which the aggregate quantity demanded across all items must increase as prices increase. For distinct items, the AMR rule is too strong for non budget-constrained bidders even in an auction with linear prices:

Example 3.2. Consider a bidder, who values a bundle with two units of item $A$ at 10 and a bundle with one unit of item B at 15. Suppose the prices are linear and the price of one unit of $A$ and one unit of $B$ in round 1 are 2 and 8 respectively and in round 2 are 3 and 12 respectively. At these prices, the bidder, when bidding straightforwardly with respect to her true values, demands the bundle with one unit of item $B$ in round 1 and two units of item A in round 2. Thus, a truthful bidder would violate the Aggregate Monotonicity Rule and be unable to express her truthful demand.

Moreover, AMR is also in a sense too weak. For example, Ausubel et al. [5] observe that a "parking" strategy is popular in FCC auctions that use AMR. In this strategy, bidders bid on many units of cheap licenses before revealing their true demand at the end of the auction. $^{11}$

\subsection{Revealed-Preference Activity Rule}

The Revealed-Preference Activity Rule (RPAR) is designed to address these difficulties with AMR [5]. To understand the rule, let package $S \subseteq \mathcal{G}$ be represented by a vector $s \in \mathbb{Z}_{\geq 0}^{|\mathcal{G}|}$, to specify the quantity of each item in the package. Let $p \in \mathbb{R}_{\geq 0}^{|\mathcal{G}|}$ denote a price vector, and

\footnotetext{
${ }^{11}$ In the FCC auction this is mitigated somewhat by defining the quantity in AMR in terms of MHzPOPs (i.e. bandwidth times population in the wireless region). This is a proxy for value, not just raw quantity, and prevents some extreme forms of strategic behavior.
} 
define a price $p_{j}$ on each item $j \in \mathcal{G}$. Then the price of package $s$, given price vector $p$, is $p \cdot s$, where $a \cdot b$ represents the inner product between two vectors.

At every round $t$ of an auction, RPAR checks that the bid $s^{t}$ of a bidder at price vector $p^{t}$ satisfies:

$$
\left(p^{t}-p^{r}\right) \cdot\left(s^{t}-s^{r}\right) \leq 0
$$

for any bid $s^{r}$ placed in an earlier round $r$ by the same bidder at price $p^{r}$ in the auction. In an auction with non-linear prices, RPAR can be easily extended to require:

$$
p^{t}\left(s^{t}\right)-p^{r}\left(s^{t}\right)-p^{t}\left(s^{r}\right)+p^{r}\left(s^{r}\right) \leq 0,
$$

which we refer to as the generalized form of RPAR.

Claim 3.3. [5] The RPAR rule is satisfied for straightforward bidders with quasi-linear utility functions in an iterative combinatorial auction, i.e. it is not too strong for such bidders.

The observation in this claim holds irrespective of whether the auction is defined for linear or non-linear prices, and resolves the problems demonstrated with MR and AMR in the earlier examples. Moreover, RPAR is in another way stronger than AMR, in that it precludes the "parking-style" manipulations [5].

However, we observe two problems with RPAR. In one sense, it is still too weak:

Claim 3.4. RPAR allows non straightforward bidding in iterative combinatorial auctions with quasi-linear utility functions and linear prices.

Proof. Proof To prove this claim, we present a counterexample in which a bidder satisfies RPAR but there exists no valuation function $v$ consistent with her bids. Consider the case when multiple units of two distinct items are being auctioned. Consider a bidder who bids the following bundles, $s^{t}$, with respect to prices $p^{t}$ : bundles $(2,0),(1,2)$ and $(0,2)$ at prices $(0,0),(1,0)$ and $(1,1)$ in rounds 1,2 and 3 respectively. Let us first check that the activity rules are satisfied:

$$
\begin{aligned}
& \left(p^{2}-p^{1}\right) \cdot\left(s^{2}-s^{1}\right)=(1,0) \cdot(-1,2)=-1 \leq 0 \\
& \left(p^{3}-p^{1}\right) \cdot\left(s^{3}-s^{1}\right)=(1,1) \cdot(-2,2)=0 \leq 0 \\
& \left(p^{3}-p^{2}\right) \cdot\left(s^{3}-s^{2}\right)=(0,1) \cdot(-1,0)=0 \leq 0 .
\end{aligned}
$$


For the other direction, assume by way of contradiction that there exists a valuation function $v$ that is consistent with the bids. Then the following is true:

$$
v\left(s^{t}\right)-p^{t} \cdot s^{t} \geq v\left(s^{r}\right)-p^{t} \cdot s^{r} \quad \text { where } r \neq t, \quad r, t=1,2,3
$$

Substituting the values of $p$ 's and $s$ 's in the above constraints we get

$$
\begin{aligned}
-1 \leq v\left(s^{3}\right)-v\left(s^{2}\right) & \leq-1 \\
-1 \leq v\left(s^{2}\right)-v\left(s^{1}\right) & \leq 0 \\
0 \leq v\left(s^{1}\right)-v\left(s^{3}\right) & \leq 0 .
\end{aligned}
$$

If we add the first and the third constraint we get $v\left(s^{2}\right)-v\left(s^{1}\right)=1$ which violates the second constraint. Hence, there exists no consistent valuation function $v$ for this bidder. This establishes our claim.

Thus, RPAR allows for some preventable bidding strategies; it is not as strong as it could be. The problem is that RPAR only ensures pairwise consistency between the bids and not a global consistency across all the bids. Thus, even if the bids satisfy RPAR, there might be no underlying utility function that "explains" (or rationalizes) the bids.

The second problem is that RPAR is actually too strong for budget-constrained bidders, and precludes straightforward bidding strategies:

Claim 3.5. A truthful, budget-constrained bidder need not satisfy RPAR, even in an auction with linear prices.

Proof. Proof Consider an auction with 2 types of items and a budget-constrained bidder with budget $\$ 5,200$ and values 7500 and 3000 for the bundles $(1,0)$ and $(0,3)$ respectively. Suppose the price vector in two successive rounds of the auction were $(\$ 5,000, \$ 500)$ and $(\$ 5,500, \$ 900)$. Clearly, if the bidder bid straightforwardly, she would bid bundle $(1,0)$ in the first round and $(0,3)$ in the second round. However, these bids violate RPAR since $(5500-5000)(0-1)+(900-500)(3-0)=700 \nless 0$.

To gain some intuition for this problem associated with RPAR when their are budgetconstraints, consider that a truthful bidder with budget $B$ places a bid $s^{r}$ in round $r$ at price 


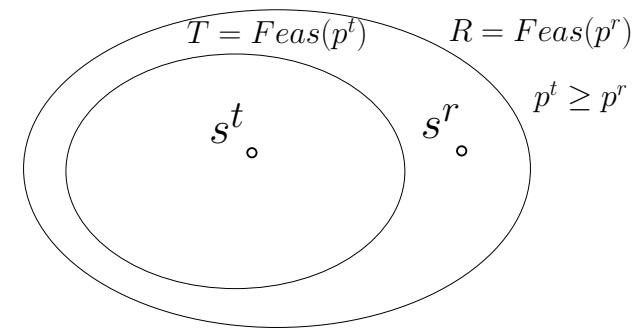

Figure 1: A scenario where RPAR might fail in the presence of budget constraints.

$p^{r}$, if

$$
v\left(s^{r}\right)-p^{r} \cdot s^{r} \geq v(s)-p^{r} \cdot s, \quad \forall s \in R=\left\{s \mid p^{r} \cdot s \leq B\right\} .
$$

Now, in a subsequent round $t$, the player bids $s^{t}$ at price $p^{t}$, if

$$
v\left(s^{t}\right)-p^{t} \cdot s^{t} \geq v(s)-p^{t} \cdot s, \quad \forall s \in T=\left\{s \mid p^{t} \cdot s \leq B\right\} .
$$

These sets, $R$ and $T$, represent the budget-feasible packages at prices $p^{r}$ and $p^{t}$ respectively. Note that because $p^{t} \geq p^{r}$, we have $R \supseteq T$. RPAR is obtained by adding the above two inequalities, with $s=s^{t}$ in the first inequality and $s=s^{r}$ in the second inequality. This step requires the fact that $s^{t} \in R$ and $s^{r} \in T$. The former is true since $R \supseteq T$. However, we can have $s^{r} \notin T$ because of budget constraints. A situation such as in Fig. 1 can happen, in which $S$ is a strict superset of $T$ leading to the failure of RPAR.

\section{Designing Strong Activity Rules and Bidder Feedback}

Having defined the concept of a strong activity rule and identified the problems with RPAR in combinatorial auctions, we will proceed to design strong activity rules and also discuss methods to provide bidder feedback. This issue of feedback is important if activity rules are to achieve the goal of promoting straightforward bidding, because the rule must be transparent enough to guide price and demand discovery.

To proceed, we will first focus on the general case of budget-constrained bidders. A strong activity rule for non budget-constrained bidders can be derived as a special case.

Consider a bidder with history $h=\left\{\left(p^{1}, S^{1}\right), \ldots,\left(p^{k}, S^{k}\right), \ldots,\left(p^{t}, S^{t}\right)\right\}$. We design a Strong Revealed Preference Activity Rule (SRPAR) by requiring that there exists a utility 
function $\theta=(v, B)$, that satisfies the following constraints:

$$
\begin{aligned}
& \text { SRPAR: } \quad v\left(S^{k}\right)-p^{k}\left(S^{k}\right) \geq v\left(S^{l}\right)-p^{k}\left(S^{l}\right) \quad \text { if } p^{k}\left(S^{l}\right) \leq B \quad \forall k, l \in\{1, \ldots, t\} \\
& p^{k}\left(S^{k}\right) \leq B, \quad \forall k \in\{1, \ldots, t\} .
\end{aligned}
$$

This places a global consistency requirement across all bids, rather than a pairwise consistency requirement as in RPAR. Constraint (4.1a) ensures that the payoff of the selected bundle $S^{k}$ for a bidder is greater than or equal to the payoff that she can achieve on other bundles $S^{l}$ at the prices, $p^{k}$, in stage $k$. The 'if' condition in (4.1a) is necessary to check if the bundle $S^{l}$ is within budget so that a valid comparison is made. Constraint (4.1b) ensures that the bundle $S^{k}$ is within budget.

We retain for now the assumption that the rules of the auction specify that only one of the maximizing bids need to be reported in each round. This is reflected in the " $\geq$ " inequality in constraint (4.1a) (as opposed to a strict ">"), when comparing the utility of the two bundles $S^{k}$ and $S^{l}$.

Theorem 4.1. SRPAR is a strong activity rule for budget-constrained bidders in iterative combinatorial auctions, both with linear and non-linear prices.

Proof. Proof Consider a consistent, budget-constrained bidder and suppose she bids according to a utility function parameterized as $\theta=\left(v_{\text {con }}, B_{\text {con }}\right)$. Thus, she behaves exactly as a truthful bidder whose $\theta=\left(v_{\text {con }}, B_{\text {con }}\right)$. That the rules will be necessarily satisfied by such a bidder is immediate, by the definition of SRPAR in constraints (4.1a-4.1b). For the sufficiency direction, consider now a bidder who satisfies the activity rule SRPAR. Suppose $\left(\left\{\widetilde{v}\left(S^{k}\right)\right\}_{k=1}^{t}, \widetilde{B}\right)$ is a feasible solution for SRPAR. We observe that if $\left(\left\{\widetilde{v}\left(S^{k}\right)\right\}_{k=1}^{t}, \widetilde{B}\right)$ is a feasible solution then so is $\left(\left\{\widetilde{v}\left(S^{k}\right)+c\right\}_{k=1}^{t}, \widetilde{B}\right)$ for fixed number $c$. Hence without loss of generality, we assume that $\widetilde{v}\left(S^{k}\right)>0$ for every $k$. Now consider a truthful bidder with budget $\widetilde{B}$ and a valuation function given as follows:

$$
v(S)= \begin{cases}\widetilde{v}\left(S^{k}\right) & \text { if } S=S^{k} \text { for some } k \in\{1, \ldots, t\} \\ 0 & \text { otherwise. }\end{cases}
$$

This truthful bidder has the same history as the original bidder, thus proving sufficiency. Note that in this case, bidders might not satisfy free-disposal. However, we can modify the 
valuation function as follows so that the bidders satisfy free disposal.

$$
v(S)=\max _{\left\{k \mid S \supseteq S^{k} ; k=1, \ldots, t\right\}}\left\{\widetilde{v}\left(S^{k}\right), 0\right\} .
$$

Clearly this valuation function satisfies free-disposal. Now if we show that $v\left(S^{k}\right)=\widetilde{v}\left(S^{k}\right)$ for all $k$, then the same proof as above proves sufficiency. Suppose this were not the case. Then it must be the case that for some $l, k$ such that $S^{k} \supset S^{l}$, we have $\widetilde{v}\left(S^{l}\right)>\widetilde{v}\left(S^{k}\right)$. Due to free-disposal, the prices satisfy $p^{r}\left(S^{k}\right) \geq p^{r}\left(S^{l}\right)$ for every round $r$. This means $\widetilde{v}\left(S^{l}\right)-p^{r}\left(S^{l}\right)>$ $\widetilde{v}\left(S^{k}\right)-p^{r}\left(S^{k}\right)$ for every round $r$ and in particular when $r=k$, we have $\widetilde{v}\left(S^{l}\right)-p^{k}\left(S^{l}\right)>$ $\widetilde{v}\left(S^{k}\right)-p^{k}\left(S^{k}\right)$. On the other hand, we have $p^{k}\left(S^{l}\right) \leq p^{k}\left(S^{k}\right) \leq B$. This contradicts that $\left(\left\{\widetilde{v}\left(S^{k}\right)\right\}_{k=1}^{t}, \widetilde{B}\right)$ is a feasible solution of SRPAR as it violates constraint (4.1a). This completes the proof.

This SRPAR rule is, of course, not too strong for non budget-constrained bidders because they are budget-constrained with an unbounded budget. On the other hand, the rule is in fact too weak for non budget-constrained bidders and will allow for deviations from straightforward bidding for such bidders.

Example 4.2. Consider a quasi-linear bidder with values 3 and 5 for two items $A$ and $B$. At prices (0,1) and (2,3), say the bidder specifies her demand as items $B$ and $A$, respectively. Then the bidder passes the rule with budget 2 and values 3 and 4 for items $A$ and $B$, respectively. But no straightforward bidder with quasi-linear utility could have this behavior because the prices have increased by the same amount on both the items.

Of course, this fact that budget-constrained bidders need to behave differently from quasilinear bidders is why RPAR was too strong for budget-constrained bidders in the first place. We return to this dilemma, wherein the appropriate rule must depend on knowledge about the domain of bidder utilities, in Section 4.2.

Implementing SRPAR. SRPAR can be rewritten as a Mixed-Integer Program (MIP) with $O\left(t^{2}\right)$ binary variables to simplify the 'if' condition in constraint (4.1a). But a rather simple observation about SRPAR simplifies its implementation.

Consider an alternate definition in which a particular budget, $\widetilde{B}$ is imposed on the feasible space of utility functions. Given this additional constraint, SRPAR is a linear feasibility 
problem with at most $O\left(t^{2}\right)$ constraints and $O(t)$ variables. Let us call this LP corresponding to a specific $\widetilde{B}$ as LP- $\widetilde{B}$. In this case, the strong activity rule would verify the existence of a feasible $v\left(S^{k}\right) \forall k \in\{1, \ldots, t\}$ by solving LP- $\widetilde{B}$. Define $B_{L}=\max _{k \in\{1, \ldots, t\}}\left\{p^{k}\left(S^{k}\right), 0\right\}$.

Observation 4.3. Consider some budget $\widetilde{B} \geq B_{L}$ and a corresponding feasible valuation function $v$ for $L P-\widetilde{B}$. Then, for any $B^{\prime} \in\left[B_{L}, \widetilde{B}\right]$, the constraints of the $L P-B^{\prime}$ are a subset of the constraints in $L P-\widetilde{B}$. So, any valuation $v$ that is feasible for $L P-\widetilde{B}$ is also feasible for $L P-B^{\prime}$.

Therefore checking SRPAR at $B_{L}$ (i.e., LP- $B_{L}$ ), which is a polynomial time check, implements the strong activity rule.

In what follows we consider three interesting variations of this strong activity rule.

Variation I: Requiring Every Best-Response Package. In the first variation, consider the design of a strong rule for an auction in which a bidder must report all utility-maximizing bundles in each round; i.e., the set of bundles across which she is indifferent.

These additional bundles are recorded in the bidder's history with higher indices but at the same price. Then the " $\geq$ " inequality that compares the utilities in constraint (4.1a) is replaced by a strict inequality when $p^{k} \neq p^{l}$, and with an equality when $p^{k}=p^{l}$ :

$$
\begin{array}{ll}
v\left(S^{k}\right)-p^{k}\left(S^{k}\right)>v\left(S^{l}\right)-p^{k}\left(S^{l}\right) \quad \text { if } p^{k} \neq p^{l} \text { and } p^{k}\left(S^{l}\right) \leq B \quad \forall k, l \in\{1, \ldots, t\} ; \text { and } \\
v\left(S^{k}\right)-p^{k}\left(S^{k}\right)=v\left(S^{l}\right)-p^{k}\left(S^{l}\right) \quad \text { if } p^{k}=p^{l} \text { and } p^{k}\left(S^{l}\right) \leq B \quad \forall k, l \in\{1, \ldots, t\} .
\end{array}
$$

For computational purposes, we can relax the strict inequality with an addition of a small number $\epsilon>0$ to the right hand side:

$$
v\left(S^{k}\right)-p^{k}\left(S^{k}\right) \geq v\left(S^{l}\right)-p^{k}\left(S^{l}\right)+\epsilon \quad \text { if } p^{k} \neq p^{l} \text { and } p^{k}\left(S^{l}\right) \leq B \quad \forall k, l \in\{1, \ldots, t\} .
$$

This activity rule can be further strengthened to ensure that the bidder also respects freedisposal. Because all utility maximizing bundles must be reported, then this also implies constraints on the utility of bundles that are not elicited. The modified activity rule is 
obtained by adding the following inequalities:

$$
\begin{array}{cl}
v\left(S^{k}\right)-p^{k}\left(S^{k}\right) \geq v(T)-p^{k}(T)+\epsilon, & \text { if } p^{k}(T) \leq B \\
& \forall T \supset S^{k}, T \neq S^{l}, p^{k}(T)>0 \forall k, l \in\{1, \ldots, t\} \\
v(T) \geq v\left(S^{k}\right), & \forall T \supset S^{k}, T \neq S^{l}, p^{k}(T)>0 \forall k, l \in\{1, \ldots, t\} .
\end{array}
$$

We introduce only as many constraints as bundles with strictly positive price, that is those bundles for which there is no subset with the same price. Thus, we avoid an exponential number of constraints except when the prices, themselves, have an exponentially-sized representation. ${ }^{12}$ This can be solved as a linear feasibility problem again using the observation 4.3. A feasible solution to this problem gives a budget, and possible values, for all the elicited bundles and their supersets. To show the rule is sufficient, one can then set the value of all other bundles (i.e., the subsets) to zero.

\section{Variation II: Quasi-Linear Bidders.}

In this second variation, we consider the special case of bidders without budget constraints. We obtain a new rule, $\mathrm{SRPAR}_{\mathrm{ql}}$, by instantiation of $B$ to infinity in SRPAR, and then through simplification. In $\mathrm{SRPAR}_{\mathrm{ql}}$, the auctioneer checks for feasibility of the following LP, where the variables are $v\left(S^{k}\right), k=1, \ldots, t$ :

$$
\operatorname{SRPAR}_{\mathrm{ql}}: v\left(S^{k}\right)-p^{k}\left(S^{k}\right) \geq v\left(S^{l}\right)-p^{k}\left(S^{l}\right), \quad \forall l, k \in\{1, \ldots, t\}, l \neq k .
$$

This constraint means the bundle $S^{k}$ placed by the bidder should be one of the utility maximizing bundles at price $p^{k}$ when compared to all other bundles, $S^{l}$, placed in the auction. This is again solvable as a linear feasibility problem, and the number of variables in the LP are $t$ and the number of constraints are $O\left(t^{2}\right)$.

We observe that $\mathrm{SRPAR}_{\mathrm{ql}}$ is a slightly enhanced (and stronger) form of RPAR, which is itself obtained by only adding constraints for every pair $l, k$ of rounds. ${ }^{13}$

We have the following corollary to Theorem 4.1 for this setting:

\footnotetext{
${ }^{12}$ In technical terms we are working, here, in the so-called exclusive-or (XOR) bidding language in which the price on a bundle is the maximal price over the price quoted on all (weak) subsets of the bundle.

${ }^{13}$ The interested reader can check that the example in Claim 3.4 which satisfied RPAR despite corresponding to an inconsistent bidder violates $\mathrm{SRPAR}_{\mathrm{ql}}$.
} 
Corollary 4.4. $S R P A R_{q l}$ is a strong activity rule for quasi-linear bidders in iterative combinatorial auctions, both with linear and non-linear prices.

Here again we assume that the rules of the auction specify that at least one (not all) of the utility maximizing bundles be placed in the auction. If the auction specifies otherwise, then we can make modifications to $\mathrm{SRPAR}_{\mathrm{ql}}$ that are directly analogous to those presented for SRPAR.

Remark 4.5. In the special case of multiple identical items, and for an auction with linear prices, then SRPAR $R_{q l}$ is equivalent to the monotonic activity rule, MR. This proves that $R P A R$, which is also equivalent to $M R$ in this case, and $M R$ are strong activity rules in this restricted environment. To see this, note that RPAR is obtained from SRPAR $R_{\text {ql }}$ by adding constraints for pair $l, k$, thus proving $S R P A R_{q l} \Rightarrow R P A R$. Now consider a bidder that satisfies RPAR i.e., $S^{k} \subseteq S^{k+1} \forall k=1, \ldots, t$. Consider a set of values $v\left(S^{k}\right), k=1, \ldots, t$ as follows:

$$
v\left(S^{k}\right)=\sum_{j=k}^{t-1} p^{j}\left[\left|S^{j}\right|-\left|S^{j+1}\right|\right]+p^{t}\left|S^{t}\right|,
$$

where $|S|$ refers to the number of items in bundle $S$. It is easy to check that these values satisfy SRPAR $R_{q l}$.

Remark 4.6. Strong activity rules can have simple forms in ascending-price combinatorial auctions with quasi-linear bidders and particular price-update rules. For example, the simple monotonicity-based activity rule in Mishra and Parkes [11], that requires that the best-response set of every bidder monotonically-increases in each round in response to price changes, is a strong activity rule for that auction.

Variation III: Relaxed Activity Rules As a third variation, we consider the possibilities for taking a strong rule as a starting point and then relaxing it somewhat.

There are a number of practical reasons for such an approach. One is that bidders may make mistakes, and a relaxed rule may be designed to provide some flexibility in order to tolerate such mistakes. Another is for reasons of complexity; this complexity concern may be both one of the computational complexity in checking the constraints, and also one of 
cognitive load on bidders. Relaxed activity rules may also be of interest because they allow bidders to adjust their values based on price feedback, for example as they make inferences about the values of other bidders. ${ }^{14}$

As a design principle, we advocate relaxing from strong rules whose properties are well understood. This will ensure that the rule that is achieved is not too strong, for example as could be the case when relaxing RPAR to handle budget-constrained bidders. In fact, by relaxing SRPAR we obtain a rule that is an appropriate relaxation of RPAR, and arguably preferable to RPAR in many practical settings because of the presence of budgets in several markets.

One interesting relaxation of SRPAR is obtained by fixing $B$ in SRPAR to $B_{L}$ and adding constraints (4.1a) for a pair of bundles $l, k$ if they both exist (i.e., they both satisfy the 'if' condition at $B_{L}$ ) or dropping them otherwise (i.e., imposing constraint (3.2) when both $p^{r}\left(S^{t}\right)$ and $p^{t}\left(S^{r}\right)$ are both less than $\left.B_{L}\right)$. The form of these constraints are similar to the constraints that define RPAR, except that they are imposed on a selected pair of bundles guided by $B_{L}$, unlike RPAR that imposes it on all pair of bundles.

Another relaxation can be obtained by allowing for approximately straightforward bidding by a bidder, wherein the bidder is within some threshold $U_{\text {error }}>0$ of its utilitymaximizing bundle in every round. In this case, we can modify SRPAR by adding an additional term $U_{\text {error }}$ on the right hand side of the constraint (4.1a). Another commonly used approach would be to provide a bidder with an opportunity to skip the rule in one or more rounds.

\subsection{Providing Bidder Feedback}

We propose some methods to generate information that can be used within a decision

\footnotetext{
${ }^{14}$ Note though, that a strong activity rule does actually already allow for some possibility in value learning. A bidder does not need to commit to a single utility function upfront. Rather, a bidder may begin bidding with some utility in mind and change this utility over time. What is required is that any change in belief about utility should be inconsequential to how she has already bid: there must exist a posteriori some particular utility to rationalize her bids across the entire auction.
} 
support tool to help to focus bidder attention on bundles that will satisfy the activity rule given earlier bids. In the case of a problem with a proposed bid, we also explain how to identify violated constraints and allow for new bids to be suggested that will satisfy the activity rule.

Feedback about Budget Bounds. One form of feedback that is useful is to provide information to a bidder about the bounds on her own budget constraint that are implied by her history of bids. We expect this to be primarily useful not because a bidder is not informed of her own budget constraint, but because a bidder may not realize the commitments that are made about her utility type through her bidding history in earlier rounds of an auction.

With an empty history, the lower bound, $B_{L}$, is initialized to 0 and the upper bound, $B_{U}$, is initialized to infinity. As the stages proceed, the tightest possible lower bound for the budget is the revealed budget i.e., $B_{L}=\max _{k \in\{1, \ldots, t\}}\left\{p^{k}\left(S^{k}\right), 0\right\}$. To understand how to compute a tight upper-bound, $B^{*} \in\left[B_{L}, B_{U}\right]$, we appeal to observation 4.3 and the general form of the SRPAR rules. By definition, this value $B^{*}$ is the supremum over all $\widetilde{B} \in\left[B_{L}, B_{U}\right]$ such that LP- $\widetilde{B}$ is feasible. Hence, clearly for all $B_{L} \leq \widetilde{B}<B^{*}$, LP- $\widetilde{B}$ is feasible and conversely for all $\widetilde{B}>B^{*}$, LP- $\widetilde{B}$ is infeasible. It turns out that $B^{*}$ is in fact the smallest budget $\widetilde{B} \in\left[B_{L}, B_{U}\right]$ such that LP- $\widetilde{B}$ is infeasible. ${ }^{15}$

In each round of the auction, as new bids are placed, more constraints are added to SRPAR. This can allow the upper-bound, $B_{U}$, to be tightened to the current $B^{*}$ and the lower-bound, $B_{L}$, to be tightened to the maximum revealed budget (See Fig. 2).

The budget bound $B^{*}$ that is implied by bids submitted in an ascending price auction

\footnotetext{
${ }^{15}$ To understand the behavior at $B^{*}$, suppose LP- $B^{*}$ is feasible. Then, consider LP- $\left(B^{*}+\delta\right)$ for a very small positive number $\delta$. LP- $B^{*}$ is the same as $\operatorname{LP}-\left(B^{*}+\delta\right)$ because $\delta$ is a very small number (more formally for small enough $\delta$ the set of constraints of LP- $B^{*}$ and LP- $\left(B^{*}+\delta\right)$ are identical $)$. Hence LP- $\left(B^{*}+\delta\right)$ is also feasible. But we know that for any $\widetilde{B}>B^{*}$, in particular when $\widetilde{B}=B^{*}+\delta$, LP- $\widetilde{B}$ does not have a feasible solution. Hence, by contradiction, LP- $B^{*}$ is infeasible.
} 


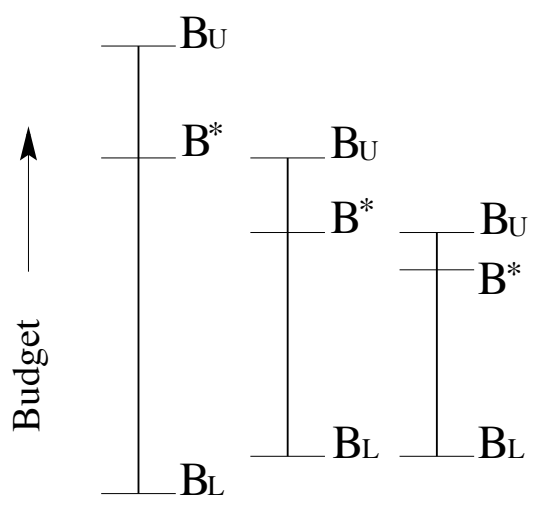

Rounds of Auction

Figure 2: The upper bound $B^{*}$ and lower-bound $B_{L}$ of a bidder's budget that is implied by its bidding behavior, versus the number of rounds of the auction.

(i.e., with $p^{k} \geq p^{l}$ for $k \geq l$ ), can be approximated by the solution to the following MIP:

$$
\begin{aligned}
& \widetilde{B_{\epsilon}}=\quad \max _{B, v\left(S^{k}\right), t_{k l}} B \\
& \text { subject to } v\left(S^{k}\right)-p^{k}\left(S^{k}\right) \geq v\left(S_{l}\right)-p^{k}\left(S^{l}\right), \quad \forall k<l, \quad k, l \in\{1, \ldots, t\} \\
& v\left(S^{k}\right)-p^{k}\left(S^{k}\right) \geq v\left(S^{l}\right)-p^{k}\left(S^{l}\right)-M t_{k l}, \quad \forall k>l, \quad k, l \in\{1, \ldots, t\} \\
& p^{k}\left(S^{l}\right) \leq B+M t_{k l}, \quad \forall k>l, \quad k, l \in\{1, \ldots, t\} \\
& p^{k}\left(S^{l}\right)+M\left(1-t_{k l}\right) \geq B+\epsilon, \quad \forall k>l, \quad k, l \in\{1, \ldots, t\} \\
& t_{k l} \in\{0,1\} \quad \forall k>l, \quad k, l \in\{1, \ldots, t\} \\
& B_{L} \leq B \leq B_{U}
\end{aligned}
$$

where $M$ is a number larger than $\max _{k, l \in\{1, \ldots, t\}} p^{k}\left(S^{l}\right)$, and $\epsilon>0$ is any small positive number.

To better understand this MIP, note that it is maximizing the budget, $B$, subject to SRPAR because the 'if' condition in constraint (4.1a) is rewritten using an indicator variable $t_{k l} \in\{0,1\}$, which is 0 if bundle $S^{l}$ at price $p^{k}$ is below budget and 1 otherwise. Considering the cases $k<l, k>l$ and $k=l$, and recalling that the auctions are ascending price constraints $(4.8 \mathrm{a}-4.8 \mathrm{e})$ are obtained. In a general demand revealing process, where the prices need not be ascending, we add constraints (4.8b-4.8e) instead of (4.8a) also in the case when $k<l$. Because of the $B_{U}$ update from the previous round, the MIP is infeasible 
for $B \geq B_{U}$. We retain the constraint $B \leq B_{U}$ for clarity.

Theorem 4.7. For iterative combinatorial auctions, a tight upper-bound $B^{*}$ on the budget constraint of a bidder that satisfies the SRPAR rule is in the range $\left(\widetilde{B_{\epsilon}}, \widetilde{B_{\epsilon}}+\epsilon\right)$.

Proof. Proof Note that $B^{*} \leq B_{U}$ because LP- $B_{U}$ is infeasible.

- $B^{*}>\widetilde{B_{\epsilon}}$ : Suppose otherwise. Then, since LP- $\widetilde{B_{\epsilon}}$ is feasible, LP- $B^{*}$ must also be feasible, contradicting the definition of $B^{*}$. Hence, $B^{*}>\widetilde{B_{\epsilon}}$.

- $B^{*}<\widetilde{B_{\epsilon}}+\epsilon$ : Suppose otherwise, and that $B^{*} \geq \widetilde{B_{\epsilon}}+\epsilon$. Consider any $B \in\left(\widetilde{B_{\epsilon}}, \widetilde{B_{\epsilon}}+\epsilon\right)$. Observe that the MIP, $P_{\epsilon}$, is feasible at $B$ as $B<B^{*}$. This contradicts that $\widetilde{B_{\epsilon}}$ is the optimal solution of $P_{\epsilon}$. Hence, $B^{*}<\widetilde{B_{\epsilon}}+\epsilon$.

To provide some intuition, it is important to understand the role of $\epsilon$ in SRPAR. In constraint (4.1a), $\epsilon$ is the small addition made to the budget, $B$, to make constraint $p^{k}\left(S^{l}\right) \leq$ $B$ feasible, which in turn introduces constraint $v\left(S^{k}\right)-p^{k}\left(S^{k}\right) \geq v\left(S^{l}\right)-p^{k}\left(S^{l}\right)$ in the LP$(B+\epsilon)$. Hence $B^{*}$ is that budget at which the introduction of a new constraint makes LP- $(B+\epsilon)$ infeasible.

This theorem defines an estimate of $B^{*}$ to within accuracy of some $\epsilon>0$, which is sufficient if $\epsilon$ is set to be smaller than the price increment between rounds. Thus, by solving this optimization problem in each round, the auctioneer not only ensures the bidder satisfies the activity rule (because the constraints of the MIP form the SRPAR) but can also compute implied bounds on the budget of the bidder.

Example 4.8. It can be easily checked that the bidder in the example provided in the proof of Claim 3.5 satisfies this new activity rule with $\widetilde{B_{\epsilon}}=5500-\epsilon$ after the second round of the auction. Hence, $B^{*} \in(5500-\epsilon, 5500)$. Thus, her budget is constrained to be between $[5000,5500)$ for all future rounds of the auction.

In fact, the value of $\widetilde{B_{\epsilon}}$ exactly lies on the price points in the auction, which suggests a simple polynomial time algorithm that the auctioneer can implement instead of solving the MIP: 
Algorithm: Sort the prices $p^{k}\left(S^{l}\right) \forall k, l \in\{1, \ldots, t\}, B_{L}$ and $B_{U}$ in ascending order (without repetitions) and retain only elements above (and including) $B_{L}$ and below (and including) $B_{U}$. Perform a binary search to find the largest element in this sorted array, $\widetilde{B_{\epsilon}}$, such that $L P-\widetilde{B_{\epsilon}}$ is feasible. If there is no such element then the MIP, $P_{\epsilon}$, is infeasible and the bidder does not satisfy the activity rules.

The worst case running time of the algorithm is equivalent to solving $O(\log t)$ linear feasibility problems.

Direct bundle feedback: In the case when the bundle submitted by the bidder does not satisfy the SRPAR activity rule, the auctioneer can suggest another bundle 'close to' the submitted bundle that satisfies the activity rule using a decision support tool. For instance, a simple decision support tool could be a linear program of finding the largest bundle that is smaller than the submitted bundle subject to the activity rule. There are many variations of this method using different distance metrics, generating multiple bundles by iterating, generating bundles subject to constraints provided by the bidder to denote 'interesting bundles' and so forth. A null bundle is always a bundle that satisfies the activity rule, along with earlier bids that also satisfied the activity rule, and hence the decision support tool can always make at least one suggestion.

\subsection{Comparing the Activity Rules}

In Table 1 we compare the different activity rules. We indicate which rules allow straightforward and truthful bidding (the necessary condition), marked by $\mathbf{T}$, and which rules are strong and also ensure consistent bidding (the necessary and sufficient condition), marked by $\mathbf{C}$. Of course we have that $(\mathbf{C} \Longrightarrow \mathbf{T})$. We mark an entry with '-' when there exists a counterexample to show the failure of $\mathbf{T}$ (and thus also $\mathbf{C}$ ). Subscript $L$ or $H$ refer to the case that the activity rule satisfies the property ( $\mathbf{T}$ or $\mathbf{C}$ ) only in the linear price setting or the homogeneous items setting respectively.

We compare across the rules in the case of a general price structure and price path and denote by $\succ$ if a rule dominates another rule, in the sense of a preference ordering in which $\mathbf{C}$ is preferred by an auction designer to $\mathbf{T}$ and $\mathbf{T}$ is preferred in turn to having neither $\mathbf{C}$ nor $\mathbf{T}$. 


\begin{tabular}{|c|c|c|c|c|c|}
\hline \multirow{2}{*}{ Utility Class } & \multicolumn{5}{|c|}{ Activity Rule } \\
\hline & MR & AMR & RPAR & $\mathrm{SRPAR}_{\mathrm{ql}}$ & SRPAR \\
\hline \multirow{2}{*}{ Quasi-Linear } & $\mathbf{C}_{L, H}$ & $\rightarrow \mathrm{C}_{L, H}$ & $\rightarrow \mathrm{C}_{L, H}$ & \multirow{2}{*}{$\mathrm{C}$} & \multirow{2}{*}{$\mathrm{T}$} \\
\hline & -1 & -- & $\mathrm{T}$ & & \\
\hline Budget-Constrained & - & - & - & $-\quad$ & C \\
\hline
\end{tabular}

Table 1: Comparison of the strength of activity rules in different settings. Arrows indicate that one rule implies another rule. Entries in bold are associated with a proof or example.

We observe that, irrespective of whether the bidders are quasi-linear or budget-constrained, RPAR dominates AMR and MR, and both SRPAR $_{\mathrm{ql}}$ and SRPAR in turn dominate RPAR, through the following reasoning:

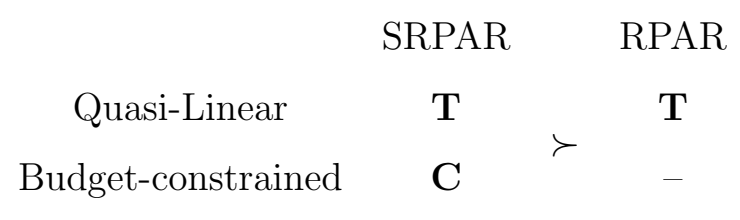

and

$\begin{array}{cccc} & \text { SRPAR }_{\mathrm{ql}} & & \mathrm{RPAR} \\ \text { Quasi-Linear } & \mathbf{C} & & \mathbf{T} \\ \text { Budget-constrained } & - & & -\end{array}$

Thus, there is an unambiguous recommendation in favor of SRPAR over RPAR. On the other hand, it is difficult to compare rules $\mathrm{SRPAR}_{\mathrm{ql}}$ and SRPAR, because SRPAR $\mathrm{Sl}_{\mathrm{q}}$ dominates SRPAR for quasi-linear bidders but is dominated by SRPAR for budget-constrained bidders.

It seems reasonable that allowing truthful bidding, $\mathbf{T}$, should receive higher priority than ensuring consistent bidding, because an activity rule should avoid "hurting" straightforward bidders by making them deviate because of the activity rule. On those lines, SRPAR is weaker than $\mathrm{SRPAR}_{\mathrm{ql}}$, and has the $\mathbf{T}$ property independent of the utility class of the bidders, 
and is likely preferred over $\mathrm{SRPAR}_{\mathrm{ql}}$ unless there is good reason to believe that there are no budget constraints.

The choice of activity rule could also be personalized to individual bidders. Of course, one would prefer an activity rule that is always strong, independent of the particular utility class of the bidders. But this is not possible. For example, a rule that allows all straightforward strategies (and is not too strong) for budget-constrained bidders must be too weak for non budget-constrained bidders. See example 4.2. On the other hand, if an activity rule is strong for non budget-constrained bidders then it must be too strong for bidders with budget constraints and preclude straightforward bidding. ${ }^{16}$ Thus, as an auction designer, it is useful to have some prior information about the types of utility functions to expect of bidders in choosing an appropriate activity rule.

\section{Experimental Simulations}

In this section, we present the results of experimental simulations that are designed to validate the importance of strong activity rules in the context of budget-constrained bidders. We study activity rules in the context of the clock-proxy auction [5]. This auction has been proposed for the landing slot auction at LaGuardia, for wireless spectrum auctions and for auctions for power generation in the context of power generation capacity [38]. We describe two variations of the clock-proxy auctions, one that incorporates RPAR and one that incorporates SRPAR.

We study the immediate problems caused when rules prevent straightforward bidding. We implement a maximally straightforward bidding strategy, such that when a deviation from straightforward bidding is necessary in order to meet the rule, we seek a simple modification to the straightforward strategy by dropping packages during the clock phase and modifying

\footnotetext{
${ }^{16}$ An easy counter-example in this scenario is as follows. Consider two bundles A and B that are being auctioned. Say in two rounds of the auction, the price of A and B increase by the same amount. Consider a bidder with values well above the prices. In the case that she is quasi-linear, the preferences should not change across the bundles. But in the case that she is budget-constrained, this behavior can be rationalized by a straightforward bidder, depending on the actual prices of the bundles and the budget.
} 
bid values associated with bids in the transition to the proxy phase. We adopt problem distributions from the CATS test suite for combinatorial auctions [39], including some of the problems originally described by Sandholm [40]. They have been widely used in the literature on combinatorial auctions $[41,42]$. A budget factor is used to assign a budget constraint to each bidder as a function of the largest value that bidder has across all packages.

\subsection{Instantiating the Clock-Proxy Auction}

The clock-proxy auction starts with a clock phase and ends with a proxy phase. The purpose of the clock phase is to provide price discovery. In each round of the clock phase the auctioneer reports linear prices and bidders respond with a package of items. Bids are XOR in nature across rounds. Prices for items with excess demand are increased and the clock phase terminates when supply is weakly greater than demand on all items. In transitioning to the proxy phase, bidders can submit a final claim about their value on every clock bundle that they have mentioned together with values on a small number $(E \geq 0)$ of additional bundles. In our context of budget constraints, we also allow bidders to make a claim about their budget constraint at this transition point.

The proxy phase is a sealed-bid auction, but simulates an ascending-price auction with non-linear and non-anonymous prices. We refer to each simulated round in this proxy phase as a proxy round. Each bidder is represented here by a proxy that follows a straightforward bidding strategy with respect to the reported valuation and budget information. This strategy is not to be confused with the maximally straightforward bidding strategy that we simulate for bidders in each actual round of the auction. This proxy bidding strategy simply defines the outcome of the final proxy phase. In our instantiation, the proxy agents bid on the set of packages in each round that maximize reported utility. A provisional allocation is computed in each proxy round to maximize revenue given bids and prices are increased to each losing bidder. The proxy phase, and thus also the entire clock-proxy auction, terminates as soon as supply is weakly greater than demand and no new bids are submitted.

We modify the behavior of the proxy phase from the standard clock-proxy auction to allow for the existence of budget-constrained bidders. Let $p_{\text {ask }}(S)$ be the ask price the auctioneer specifies for bundle $S$. For bundle $S$ where $p_{\text {ask }}(S) \leq B$ and $p_{\text {ask }}(S) \leq v(S)$, the 
proxy bid price, $p_{\text {bid }}(S)$, is simply $p_{\text {ask }}(S)$. For bundles with $p_{\text {ask }}(S)>B$ or $p_{\text {ask }}(S)>v(S)$, the bid price is $p_{\text {bid }}(S)=p_{\text {ask }}(S)-\epsilon$, where, $\epsilon>0$ is the bid increment in the proxy stage. By adopting this " $\epsilon$-discount" the effective ask prices on these bundles does not increase further.

A proxy agent reports packages in each round that (a) maximizes the quasi-linear utility (value minus price) to within $\epsilon$ among all those bundles that are priced below value and below budget or priced just above the value (b) has quasi-linear utility at least as large as that in (a) but priced just above budget. This bidding strategy ensures that the proxy's demand set is monotonically increasing across rounds. Because of the price dynamics, this has the effect of reporting packages, $S \subseteq \mathcal{G}$, in each round that satisfy:

$$
v(S)-p_{\text {bid }}(S)+\epsilon \geq \max _{T \in \mathcal{G}_{B}}\left\{v(T)-p_{\text {bid }}(T)\right\},
$$

where the maximum is taken over a restricted set of bundles,

$$
\mathcal{G}_{B}=\left\{T \subseteq \mathcal{G} \mid\left[v(T) \geq p_{\text {ask }}(T) \text { and } p_{\text {ask }}(T) \leq B\right] \text { or }\left[v(T)<p_{\text {ask }}(T)\right]\right\}
$$

We now provide details of the application of SRPAR and RPAR to the clock proxy auction, including a description of the impact of the activity rule on our construction for a maximally-straightforward bidding strategy.

The Clock-Proxy Variation with SRPAR. In the clock stage we implement SRPAR exactly as described in Section 4. At the transition to proxy we impose SRPAR directly for the clock bundles along with some additional constraints for the extra transition bundles. A bidder can report up to $E \geq 0$ additional bundles that were not mentioned in the clock stage (the "clock bundles"), and for these bundles and the clock bundles, associate a valuation function $\hat{v}$ that together with a claim about a budget constraint $\hat{B}$ (perhaps infinite), provides a feasible solution to the SRPAR constraints in (4.1) along with the following constraints:

$$
\begin{array}{rlrl}
v\left(S^{k}\right)-p^{k}\left(S^{k}\right) & \geq v(U)-p^{k}(U) \quad \text { if } p^{k}(U) \leq B \quad \forall S^{k} \in \text { Bids }_{\text {clock }}, U \in \text { Bundles }_{\text {new }} \\
v(U) & \geq v(S) \\
\forall U \supset S, S \in \text { Bundles }_{\text {clock }}, U \in \text { Bundles }_{\text {new }},
\end{array}
$$

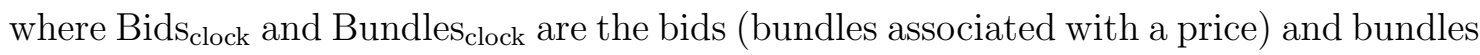
elicited in the clock stage respectively and Bundles ${ }_{\text {new }}$ are the additional transition bundles. 
Constraint (5.3) is the same as constraint (4.1a) in SRPAR. Constraint (5.4) just ensures free-disposal.

The straightforward bidding strategy for budget-constrained bidders in clockproxy with SRPAR is defined as:

(a) Clock stage: In each round, bid on the package that maximizes utility given a valuation function and budget constraint, and given current prices.

(b) Transition stage: Select the additional $E \geq 0$ bundles that maximize quasi-linear utility, given the final clock prices and ignoring the budget constraints. Report the true value for all clock bundles and all additional bundles together with the true budget constraint information.

The Clock-Proxy Variation with RPAR. In the clock stage we implement RPAR exactly as described in Sections 3. We also need an activity rule at the transition from from clock to proxy. For RPAR, we adopt a relaxed rule that is inspired in part by some of the operational details in Hoffman [43], while differing in substance in order to better allow for budget-constrained bidders during the clock stage ${ }^{17,18}$. The relaxed RPAR at the transition is parameterized by relaxation parameter, $\alpha>1$, and defined as:

$$
\begin{aligned}
\alpha\left[v\left(S^{k}\right)-p^{k}\left(S^{k}\right)\right] & \geq v\left(S^{l}\right)-p^{k}\left(S^{l}\right) & & \forall S^{k}, S^{l} \in \text { Bids }_{\text {clock }} \\
\alpha\left[v\left(S^{k}\right)-p^{k}\left(S^{k}\right)\right] & \geq v(U)-p^{k}(U) & & \forall S^{k} \in \text { Bids }_{\text {clock }}, U \in \text { Bundles }_{\text {new }} \\
p^{\max }(S) & \leq v(S) & & \forall S \in \text { Bundles }_{\text {clock }},
\end{aligned}
$$

\footnotetext{
${ }^{17}$ Hoffman [43] specified an upper bound on the values of clock as well as the new bundles based on their prices in the final rounds for a drop-out bidder. But we cannot upper bound the true value for budgetconstrained bidders and hence do not impose these constraints. Furthermore, these authors also suggested to include a lower bound on the values of new bundles based on prices. We do not include this constraint because the prices at the end of clock phase need not be representative of the prices at the end of the proxy stage with budget-constrained bidders.

${ }^{18}$ Ausubel et al. [5] also suggest the use of a relaxed activity rule, but for a different reason. Their concern is to address demand reduction in the clock phase, which can occur because of linear pricing. A relaxed activity rule allows bidders to reverse some of this demand reduction.
} 
where $p^{\max }(S)$ is the maximum price that the bidder has bid on bundle $S$ during the clock stage. This activity rule ensures two things. Firstly it ensures that the clock bundles maximize the quasi-linear utility with an $\alpha$ relaxation with respect to any other clock bundle and that they are valued higher than the maximum bid price on that bundle. Secondly it ensures that any transition bundle has lower quasi-linear utility than a clock bundle with a $\alpha$ relaxation.

In defining a bidding strategy for budget-constrained bidders in clock-proxy with RPAR rather than SRPAR we adopt as close an approximation to a straightforward, truthful bidding strategy as is possible given the activity rule.

The following example shows that a bidder might be unable to meet RPAR at the transition to proxy, even without trying to submit additional bundles and for some $\alpha>1$.

Claim 5.1. A truthful budget-constrained bidder may be unable to meet the relaxed RPAR rule in the transition from clock to proxy by simply associating truthful values with each of the clock bundles, even when the relaxation parameter, $\alpha$, exceeds 1 .

Proof. Proof It suffices to provide an example of a scenario where this happens. We provide an example in the case when $\alpha=1.05$. Consider an auction of two items A and B. Consider a bidder with a budget of 100 whose value for item $\mathrm{A}$ is 125 and whose value for item $\mathrm{B}$ is 110. Say the prices in the last two rounds of the auction are as shown in the following table. Also, shown are the packages, $x^{t}$, that form the best-response of the bidder:

$\begin{array}{ccc}\text { Round } & p^{t} & x^{t} \\ \mathrm{t}-1 & (80,75) & (1,0) \\ \mathrm{t} & (110,100) & (0,1)\end{array}$

These two bundles satisfy RPAR because $(110-80)(0-1)+(100-75)(1-0)=-5<0$. However, RPAR constraint (5.5) for $\alpha=1.05$, written in terms of the valuations reported at this transition from clock to proxy, requires $1.05(v(A)-80) \geq v(B)-75$ and $1.05(v(B)-$ $100) \geq v(A)-110$. Valuations, $v(A)=125$ and $v(B)=110$, cause the second inequality to be violated. It is also interesting to observe that for $\alpha=1$, the constraints are always infeasible for any valuations. 
So, bidders can be forced to report false values on clock bundles at the transition, and sometimes there can be no reports that will satisfy RPAR! To overcome this difficulty we further relax $\alpha$-RPAR at the transition, by always allowing every bundle in the clock phase to be submitted with a value no less than the maximum price bid on the bundle in the clock phase. This allows all bundles that receive a bid in the clock stage to be passed on to the proxy stage.

The maximally straightforward bidding strategy for budget-constrained bidders in clock-proxy with RPAR is defined as:

(a) Clock stage: In each round, greedily pick a utility maximizing bundle (by definition, within budget) at the current prices satisfying RPAR.

(b) Transition stage:

(b1) Select a subset of the clock bundles that will satisfy $\alpha$-RPAR at values discounted from the true values and to minimize the maximum difference of the submitted values from the true values. We formulate this as a mathematical program described in the Appendix. All other bundles are submitted at the maximal bid price from the clock phase.

(b2) Sort the remaining (non-clock) bundles in order of decreasing quasi-linear utility at the final clock prices, and greedily pick the first $E \geq 0$ packages (if any) that satisfy $\alpha$-RPAR when associated with the bidder's true value and with the values already assigned in step (b1) to clock bundles. The budget information is not considered in picking these extra bundles.

\subsection{Defining an Efficiency Metric for Budget-Constrained Bidders}

For quasi-linear utilities, an efficient allocation is defined as the one that maximizes the total value of the allocation across the bidders. Although one can also adopt the same definition of efficiency for budget-constrained bidders, this benchmark is more problematic in this context because it is often unattainable when coupled with strategic bidders; e.g. the VCG mechanmism fails (see Borgs et al. [28], Dobzinski et al. [29]). 
Consider a single item auction with two bidders, A and B. Say A and B have values of 10 and 8 for the item with budgets of 4 and 6 respectively. The maximum value allocation cannot be supported as a price equilibrium as B has sufficient budget to outbid A. Consider also limiting case of budget constraints $B=0$ for every bidder with multiple items. Now, because payments cannot be collected from bidders, this reduces to the setting of voting theory. The Gibbard-Satterthwaite theorem [44, 45] states that any strategy-proof voting rule is dictatorial if there are at least three outcomes in the range of the rule. Thus, Pareto efficiency, but not allocative efficiency in the sense of maximizing total value, can be achieved.

An alternative metric, that is simple and somewhat intuitive, is to define the efficiency of an allocation $S^{*}$ in terms of the sum, over all bidders, of the minimum of the value and the budget $\left(\min \left\{v\left(S_{i}^{*}\right), B\right\}\right)$ for each bidder [28]. The best allocation is that which maximizes the sum of this "min $(v, B)$ value" over all bidders. The intuition behind the metric is that it will be hard to effect tradeoffs between the value of one bidder and another when these values are greater than the budgets of the bidders because prices cannot be used to effect the tradeoff. Indeed, this is achievable in the context of mechanism design for a single-item setting ${ }^{19}$. We choose to adopt both the total value and this total $\min (v, B)$-value in presenting our results. This latter metric is also used to provide a normalization when reporting the revenue achieved in the clock-proxy auction in the presence of budget constraints ${ }^{20}$.

\footnotetext{
${ }^{19}$ It is unknown whether or not the target of the allocation that maximizes this $\min (v, B)$ value is achievable in the combinatorial setting. Maskin [26] elaborates issues with regard to the definition of efficiency for a single item and suggests a new definition called "constrained efficiency" in an all-pay single item auction setting. Its extension to multiple items, however appears to be an open problem.

${ }^{20}$ One can also consider a metric defined in terms of an approximate budget-constrained price equilibrium, where $S^{*}$ and prices $p^{*}$ are in an equilibrium when: (a) the allocation (approximately) maximizes each bidder's utility at the prices and the bidder's (given) budget constraint; and (b) the allocation (approximately) maximizes the seller's revenue at the prices. Given this allocation, the efficient allocation would be defined to maximize the total value $v(S)$ over all bidders, across all allocations $S$ for which there exists an approximate, budget-constrained price equilibrium. While this can be formulated as an enormous MIP, we have been unable to find an operational methodology to compute this benchmark.
} 


\subsection{Experimental Results}

The distributions on bidder valuations that we adopt in our experiments are the arbitrary, matching, paths and scheduling distributions and the two legacy distributions (L2 - uniform with linearly random and $L 4$ - decay with linear random) [40]. For each distribution we generate 20 instances and present our results averaged over these instances. We choose distribution-specific parameters (such as maxbid) so that on average each bidder has a value for at least 10 bundles and adopt the exclusive-or (XOR) valuation logic, so that the bidder's value for some bundle $S$ is the maximal value over all bundles that are a subset of $S$. Having valuation functions with at least 10 bundles makes them sufficiently complex so that the distinction between RPAR and SRPAR matters. For the legacy distributions, we follow Parkes and Ungar [9] and generate valuations on bundles for each bidder independently, and join them together to form the input to our auction simulator.

We were unable to adjust the parameter in paths to achieve suitably large valuation functions (bidders valued on average only up to 3 bundles even with tuned parameters). Because of this the results for the paths distribution are the same for RPAR and SRPAR (as RPAR and SRPAR are equivalent in the limiting case of one bundle)and are not presented here.

To analyze the efficiency and revenue results for various budgets, we introduce a notion called the budget factor: the budget factor $B F \in(0,1]$, defines the budget $B$ of a bidder as a fraction $B F$ of the maximum value that the bidder has over all bundles. In our experiments, we make the simplifying assumption that all bidders have the same budget factor. We compute the following efficiency and revenue metrics:

$\mathrm{VB} / \max \mathrm{VB}$ - Ratio of the total $\min \{v(S), B\}$ of the allocation resulting from the auction to the maximum total $\min \{v(S), B\}$ over all allocations.

$\mathrm{CP} / \max \mathrm{V}$ - Ratio of the total value of the allocation resulting from the auction to the maximum total value over all allocations (the traditional definition of efficiency)

$\mathrm{R} / \mathrm{maxVB}$ - The ratio of the revenue in the auction to the maximum of the total $\min \{v(S), B\}$ over all allocations. We normalize the revenue by the maximum of the 
total $\min \{v(S), B\}$ because this represents the maximum transferable monetary value from the bidders to the auctioneer at the specified budgets.

We vary budget factor $B F$, the number of extra bundles $E \geq 0$ that can be submitted upon transition to the proxy phase, and the $\alpha \geq 1$ relaxation to RPAR. We adopt the following notation in the figures:

$B F$ - Budget factor

$E$ - Number of extra bundles at transition

$A$ - The relaxation parameter $\alpha$ for clock-proxy with RPAR

New - The performance of clock-proxy with SRPAR, our proposed new activity rules

Old - The performance of clock-proxy with RPAR, the standard revealed-preference activity rule and as relaxed by parameter $\alpha \geq 1$.

Figures 3- 7 illustrate the results for the L4 (decay) legacy, L2 (uniform) legacy, matching arbitrary and scheduling distributions. In each of these figures we vary the budget factor $B F$. Subplot $(a)$ reports the $\min \{v, B\}$ efficiency metric $(\mathrm{VB} / \max \mathrm{VB}),(b)$ reports the traditional efficiency metric $(\mathrm{CP} / \mathrm{maxV})$, and $(c)$ reports the revenue metric $(\mathrm{R} / \mathrm{maxVB})$. These subplots fix $E=4$ and consider both $\alpha=1.05$ and $\alpha=1.0$ for RPAR. We have done experiments for several values of the parameters and observed a qualitatively similar trend across the parameters.

One general observation based on the results in the figures is that SRPAR tends to dominate the performance of RPAR with respect to the $\min \{v, B\}$ efficiency (subplots $(a)$ ) and revenue metrics (subplots $(c)$ ). In general, we observe a maximum improvement at low budget factors (3.8\% and $7.8 \%$ on average) and qualitatively less at high budget factors. The amount of improvement varies with distribution and is as high as $13 \%$ and $20 \%$ for the L4 legacy distribution (averaged over BFs 0.1 and 0.2) to an insignificant difference for the arbitrary distribution.

Even though we present the traditional efficiency metric, $\mathrm{CP} / \max \mathrm{V}$, in subplots $(b)$, this metric is less meaningful when budgets are constrained because of the issues discussed in 
Section 5.2. As can be seen in the plots, although there is a marginal improvement for L4, L2 and arbitrary distributions, it marginally under performs for the matching and scheduling distributions.

While the performance of clock-proxy with respect to the efficiency metrics does not strictly improve with the budget factor, the performance generally improves with increasing $B F$ for both RPAR and SRPAR. The revenue metric has a decreasing trend with budget factor in the matching distribution and an increasing trend in other distributions. The decreasing trend should not be a surprise because the goal of the clock-proxy auction is to maximize efficiency rather than revenue and it could very well be the case that they occur at different auction outcomes.

We have also considered the effect of varying the number of extra bundles, $E$, that can be submitted at the transition to proxy. We find that the $\min \{v, B\}$ efficiency and revenue metrics improve with $E$, in return for allowing more bids to be submitted to the proxy. This is not the case for the max $V$ efficiency metric, possibly again because of the discrepancy in the choice of this metric for budget constrained bidders.

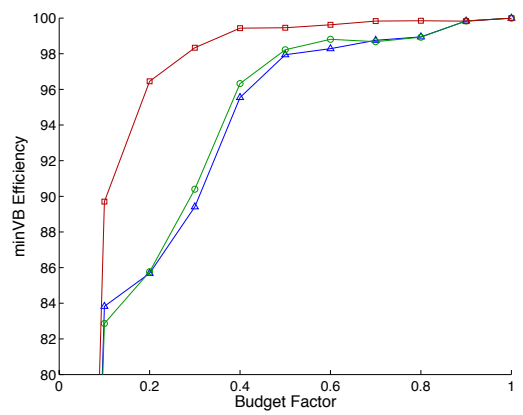

(a)

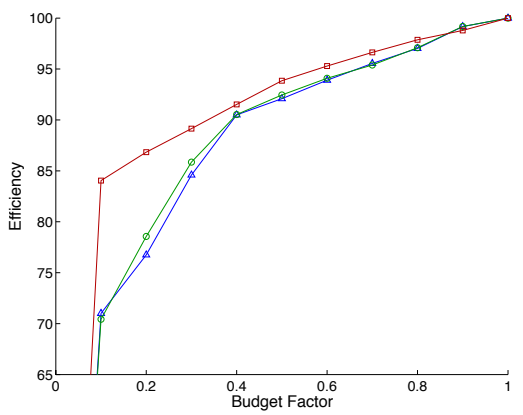

(b)

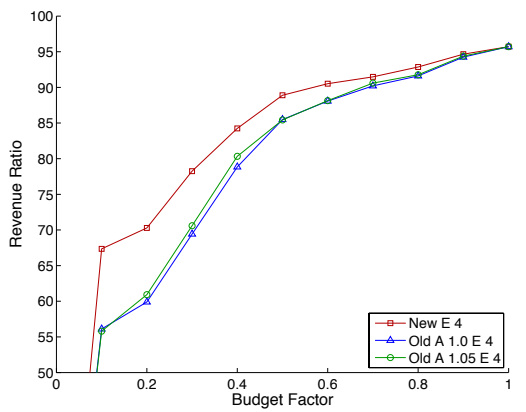

(c)

Figure 3: L4 Legacy - Decay and Linearly Random - 20 items and 20 bidders with 12.5 bundles per bidder.

Turning to the relaxation parameter $\alpha$, and clock-proxy with RPAR, we see from the subplots that the performance with $\alpha=1.05$, a more relaxed rule, tends to dominate that for $\alpha=1.0$, in general, but we do observe the reverse as well. The reason why such a behavior is possible is because we are working with a hybrid auction where (a) there is loss in information during the transition from clock to proxy and (b) the transitioning rules in the case of RPAR does not lead to submitting (i) true values for the clock bundles (but close 


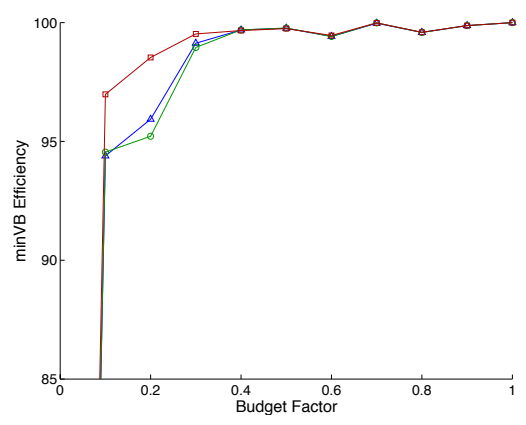

(a)

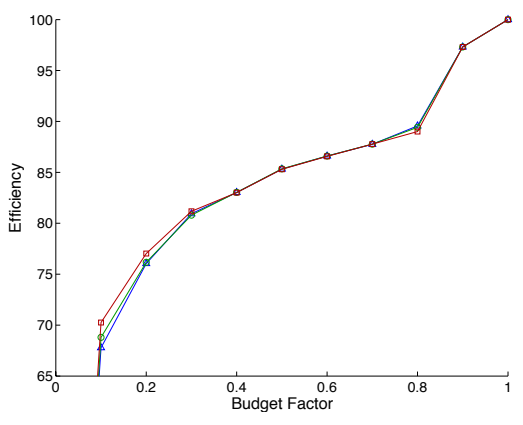

(b)

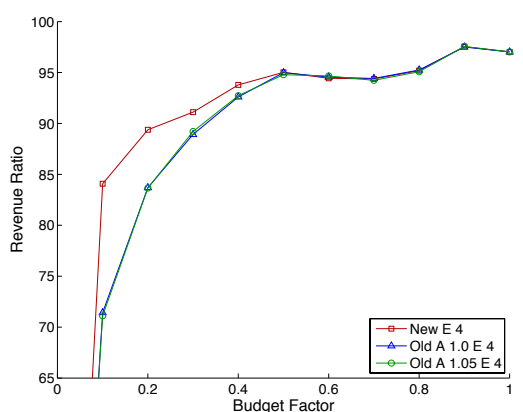

$(c)$

Figure 4: L2 Legacy - Uniform and Linearly Random - 20 items and 20 bidders with 15 bundles per bidder.

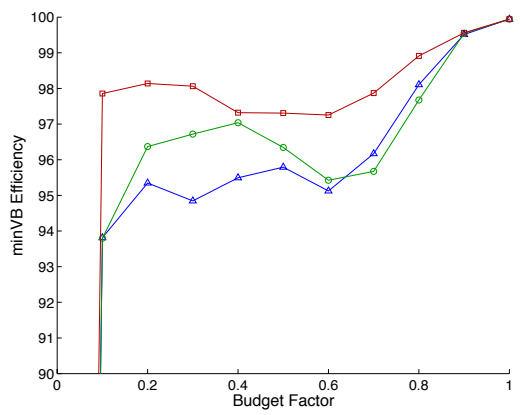

(a)

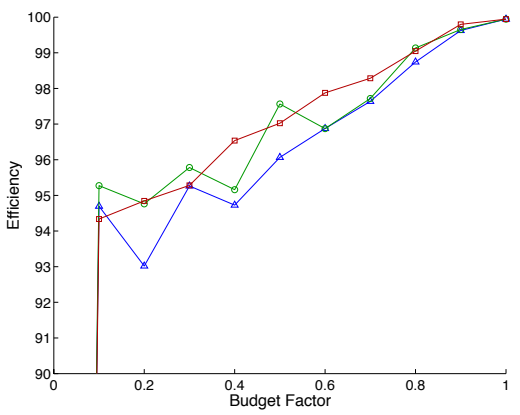

(b)

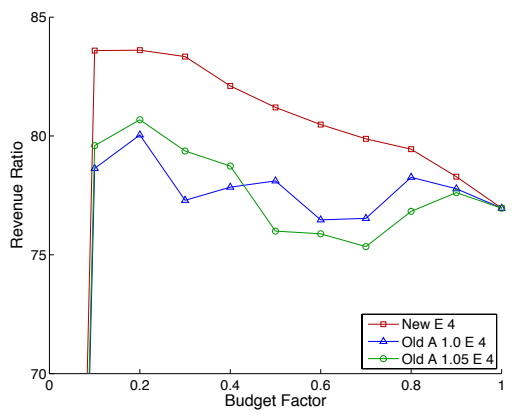

(c)

Figure 5: CATS - Matching - 32 items and total number of bundles randomly chosen between 400-500 (resulting in average of 13.3 bundles per bidder and 34 bidders).

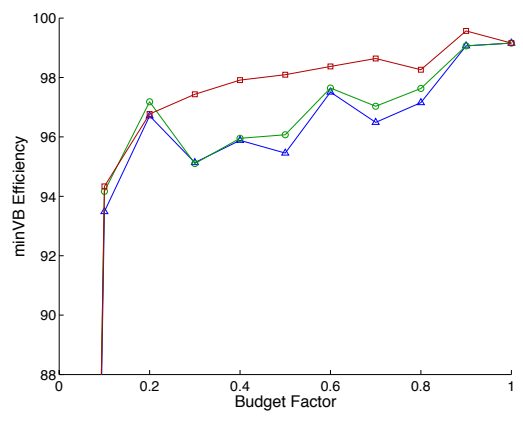

(a)

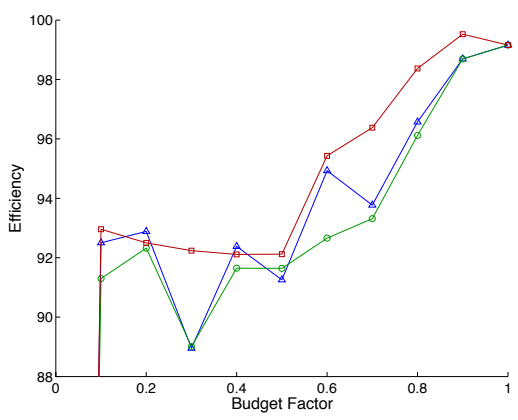

(b)

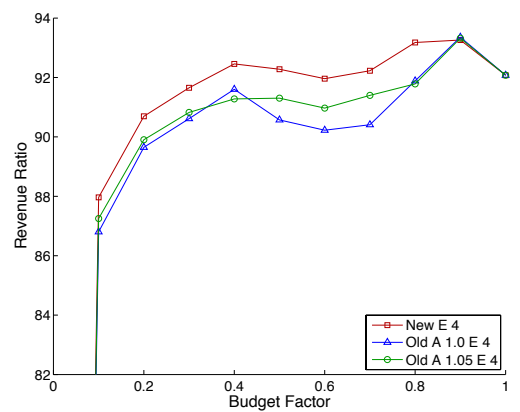

(c)

Figure 6: CATS - Arbitrary - 25 items and total number bundles randomly chosen between 400-500 (resulting in an average of 8.4 bundles per bidder and 26 bidders).

to true values) and (ii) more bundles for the relaxed rule as compared to the tighter rule as one might expect. See the clock-proxy variation with RPAR in Section 5.1. 


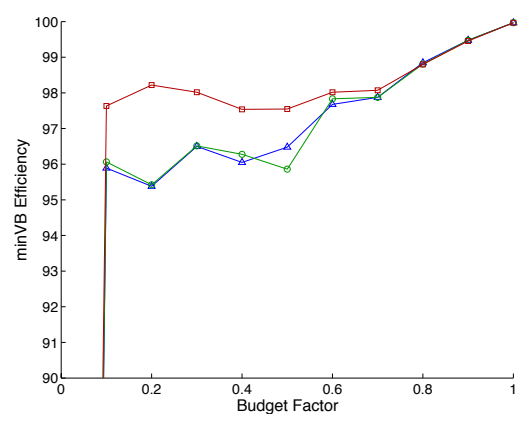

(a)

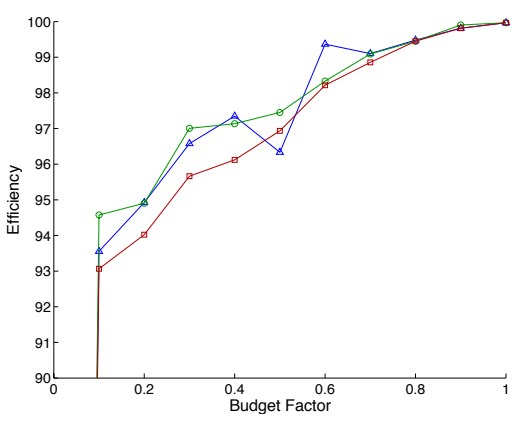

(b)

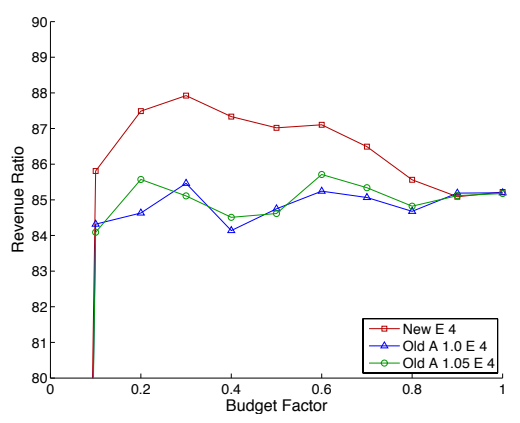

(c)

Figure 7: CATS - Scheduling - 25 items and total number of bundles randomly chosen between 200-400 (resulting in average of 18.6 bundles per bidder and 18 bidders.)

Additional evidence that we observed in our experiments, and also suggestive of better performance with SRPAR than RPAR, is that the number of rounds in the clock auction with SRPAR tends to be greater than with RPAR. This is because not all straightforward bundles can pass RPAR. We also observe that the set of bundles submitted to the proxy in clock-proxy with SRPAR tends to be a superset of the bundles submitted by RPAR.

\section{Concluding Remarks}

Activity rules are important in practical auction design because they promote price discovery through simple, consistent bidding by auction participants. We have advocated the use of strong activity rules, and developed such a rule for budget-constrained bidders. As a special case for non budget-constrained bidders, we recover a rule that strengthens the well known revealed-preference activity rule and prevents some undesirable behaviors. While the strong activity rule for budget-constrained bidders allows some inconsistent bidding in the presence of non budget-constrained bidders, it nevertheless allows simple, consistent bidding for such bidders and is likely to be useful in practice.

The proposed strong activity rules provide an improvement in the efficiency and revenue properties of the clock-proxy auction in experiments, when populated with maximally straightforward bidders. The relative performance from SRPAR over RPAR improves for tighter budget constraints. 


\section{Acknowledgements}

We thank Peter Cramton for providing ideas on direct feedback methods and Peter Belobaba for useful discussions on the state of the airline industry. We are grateful to the anonymous reviews for their constructive comments about an earlier version of this paper. The first author would like to thank Prahladh Harsha for several useful comments on the presentation of the paper. Thanks to Sébastien Lahaie for providing a code base that we adapted for simulations, results of which are in an extended version of this work. This work is informed also by Rui Dong's early work in her senior thesis at Harvard University 2005.

\section{A. Computing the Bidding Strategy in Clock-Proxy with RPAR.}

We formulate the problem of assigning values to clock bundles, i.e. bundles already mentioned in the clock stage, while meeting RPAR at the transition from clock to proxy. The problem is to pick a subset of the clock bundles that will satisfy RPAR at values that are minimally discounted from true values. We break ties based on the total submitted value of the bundles. We formulate this problem as a MIP:

$$
\begin{array}{rlrl}
\min M \delta-\epsilon \sum_{k \in D} \hat{v}\left(S^{k}\right) & & \\
v\left(S^{k}\right)-\hat{v}\left(S^{k}\right) & \leq \delta & & \\
\hat{v}\left(S^{k}\right) & =\hat{v}\left(S^{l}\right) & & \forall k \in T \\
p^{\max }\left(S^{k}\right) z_{k} & \leq \hat{v}\left(S^{k}\right) \leq v\left(S^{k}\right) z_{k} & & \\
z_{k}+z_{l} & =2-y_{k l} & & \forall l, k(\neq l) \in T \\
M y_{k l}+\alpha\left[\hat{v}\left(S^{k}\right)-p^{k}\left(S^{k}\right)\right] & \geq \hat{v}\left(S^{l}\right)-p^{k}\left(S^{l}\right) & & \forall l, k(\neq l) \in T \\
z_{k} & \in\{0,1\} & & \forall l, k(\neq l) \in T \\
y_{k l} & \in\{0,1,2\} & & \forall l, k(\neq l) \in T \\
\delta & \geq 0 & &
\end{array}
$$

where $T=\{1, \ldots, t\}$ and $D=\left\{k \mid S^{k} \neq S^{l}, \forall k, l \in T\right\}$, so that all bundles are accounted only once in the objective. This is necessary because a bundle could be elicited several times in 
the clock auction. Value $\hat{v}\left(S^{k}\right)$ is the submitted value of bundle $S^{k}$ whose true value is $v\left(S^{k}\right)$. Constant, $M>0$ is a large number and is set equal to $10 * \max _{k \in T} v\left(S^{k}\right)$. Constant, $\epsilon>0$ is a small positive number. Variable, $\delta$ is the maximum difference between the true value and the submitted value. Since $\delta$ is constrained to be non-negative, the submitted value is always lower than the true value. This constraint is particularly critical when the value of a bundle is lower than the budget. The objective is hybrid, in the sense that it first minimizes $\delta$ and then breaks ties based on the total submitted value. Constraint (A.1c) ensures that the values of two bundles elicited in different rounds of the auction are the same as long as the bundles are the same. $z_{k} \in\{0,1\}$ is a variable that is set to 1 if the bundle $S^{k}$ is chosen by the MIP, and 0 otherwise. Variable $y_{k l} \in\{0,1\}$ is set to 0 only if both variables $z_{k}$ and $z_{l}$ are 1 and is used to enforce the activity rule between the pair of bundles $S^{k}$ and $S^{l}$. Constraint (A.1f) checks that the activity rule is satisfied when both bundles $S^{k}$ and $S^{l}$ are chosen by the MIP.

\section{References}

[1] P. Cramton, Y. Shoham, R. Steinberg (Eds.), Combinatorial Auctions, MIT Press, 2006.

[2] D. C. Parkes, Iterative Combinatorial Auctions, in: [1], chap. 2, 2006.

[3] P. C. Cramton, Money Out of Thin Air: The Nationwide Narrowband PCS Auction, Journal of Economics and Management Strategy 4 (1995) 267-343.

[4] M. O. Ball, G. Donohue, K. Hoffman, Auctions for the safe, efficient and equitable allocation of airspace resources, in: [1], chap. 20, 2006.

[5] L. M. Ausubel, P. Cramton, P. Milgrom, The Clock-Proxy Auction: A Practical Combinatorial Auction Design, in: [1], chap. 5, 2006.

[6] P. Milgrom, Putting Auction Theory to Work: The Simultaneous Ascending Auction, Journal of Political Economy 108 (2000) 245-272.

[7] R. Wilson, Activity Rules for an Iterated Double Auction, in: W. F. Samuelson, 
K. Chatterjee (Eds.), Game Theory and Business Applications, chap. 12, Springer, 2001.

[8] L. M. Ausubel, P. Milgrom, Ascending Auctions with Package Bidding, Frontiers of Theoretical Economics 1 (1) (2002) 1-42.

[9] D. C. Parkes, L. H. Ungar, Iterative Combinatorial Auctions: Theory and Practice, in: Proc. of the National Conference on Artificial Intelligence, 74-81, 2000.

[10] L. M. Ausubel, An Efficient Ascending-Bid Auction for Multiple Objects, American Economic Review 94 (5) (2004) 1452-1475.

[11] D. Mishra, D. C. Parkes, Ascending Price Vickrey Auctions for General valuations, Journal of Economic Theory 127 (1) (2007) 335-366.

[12] S. de Vries, J. Schummer, R. V. Vohra, On ascending Vickrey auctions for heterogeneous objects, Journal of Economic Theory 127 (1) (2007) 95-118.

[13] P. Cramton, The Revealed Preference Activity Rule, (Working Paper), 2007.

[14] M. O. Ball, L. M. Ausubel, F. Berardino, G. Donohue, M. Hansen, K. Hoffman, MarketBased Alternatives for Managing Congestion at New Yorks LaGuardia Airport, in: Proc. of AirNeth Annual Conference, 2007.

[15] ATA, Outlook: Reaching for the skies?, Air Transport Association, 2007.

[16] P. Belobaba, Impacts of 9/11 on US Airline Performance, in: MIT Research and Development Conference, 2005.

[17] D. J. Salant, Up in the air:GTE's experience in the MTA auction for personal communication services licenses, Journal of Economics and Management Strategy 6 (3) (1997) $549-572$.

[18] C. Pitchik, Budget-Constrained Sequential Auctions with Incomplete Information, (Working Paper), 2006. 
[19] Y.-K. Che, I. Gale, Standard Auctions with Financially Constrained Bidders, Review of Economic Studies 65 (1) (1998) 1-21.

[20] D. Porter, S. Rassenti, A. Roopnarine, V. Smith, Combinatorial Auction Design, Proc. of the National Academy of Sciences 100 (10) (2003) 11153-11157.

[21] L. M. Ausubel, An Efficient Dynamic Auction for Heterogeneous, American Economic Review 96 (3) (2006) 602-629.

[22] A. M. Kwasnica, J. O. Ledyard, D. Porter, C. DeMartini, A New and Improved Design for Multiobject Iterative Auctions, Management Science 51 (3) (2005) 419-434.

[23] P. R. Wurman, M. P. Wellman, AkBA: A progressive, anonymous-price combinatorial auction, in: ACM Conference on Electronic Commerce, 21-29, 2000.

[24] J.-J. Laffont, J. Robert, Optimal auctions with financially constrained buyers, Economic Letters 52 (1996) 181-186.

[25] Y.-K. Che, I. Gale, The optimal mechanism for selling to a budget-constrained buyer, Journal of Economic Theory 92 (2000) 198-233.

[26] E. S. Maskin, Auctions, development and privatization: Efficient auctions with liquidityconstrained bidders, European Economic Review 44 (2000) 667-681.

[27] J.-P. Benoit, V. Krishna, Multiple-Object Auctions with Budget-Constrained Bidders, Refiew of Economic Studies 68 (2001) 155-179.

[28] C. Borgs, J. Chayes, N. Immorlica, M. Mahdian, A. Saberi, Multi-unit auctions with budget-constrained bidders, in: Proc. of the ACM conference on Electronic commerce, $44-51,2005$.

[29] S. Dobzinski, R. Lavi, N. Nisan, Multi-unit Auctions with Budget Limits, in: Proc. of 49th Annual IEEE Symposium on Foundations of Computer Science, 260-269, 2008.

[30] M. M. Pai, R. Vohra, Optimal Auctions with Financially Constrained Bidders, Tech. Rep., Kellogg School of Management, Northwestern University, 2008. 
[31] G. Aggarwal, S. Muthukrishnan, D. Pal, M. Pal, General Auction Mechanism for Search Advertising, Tech. Rep. CoRR abs/0807.1297, Google, 2008.

[32] L. M. Ausubel, P. Milgrom, Ascending Proxy Auctions, in: [1], chap. 3, 2006.

[33] J. Kornai, E. Maskin, G. Roland, Understanding the Soft Budget Constraint, Journal of Economic Literature 41 (4) (2003) 1095-1136.

[34] S. N. Afriat, The Construction of Utility Functions from Expenditure Data, International Economic Review 8 (1) (1967) 67-77.

[35] R. Vohra, Paths, Cycles and Mechanism Design, Tech. Rep., Kellogg School of Management, 2007.

[36] R. W. Day, A Revealed-Preference Activity Rule for Quasi-Linear Utilities with Budget Constraints, (Working Paper), 2007.

[37] P. Cramton, Ascending Auctions, European Economic Review 42 (3-5) (1998) 745-756.

[38] P. Cramton, S. Stoft, Colombia Firm Energy Market, in: Proc. of the Hawaii International Conference on System Sciences, 2007.

[39] K. Leyton-Brown, Y. Shoham, A Test Suite for Combinatorial Auctions, in: [1], chap. 18, 2006.

[40] T. Sandholm, Algorithm for optimal winner determination in combinatorial auctions, Artificial Intelligence 135 (1-2) (2002) 1-54.

[41] T. Sandholm, S. Suri, A. Gilpin, D. Levine, CABOB: a fast optimal algorithm for winner determination in combinatorial auctions, Management Science 51 (3) (2005) 374-391.

[42] A. Pikovsky, P. Shabalin, M. Bichler, Iterative Combinatorial Auctions with Linear Prices: Results of Numerical Experiments, in: Proc. of the 8th IEEE international conference on E-Commerce Technology, vol. 0, 39-39, 2006.

[43] K. Hoffman, Hybrid Clock Proxy Auction - Implementation Details, (Private Correspondence), 2004. 
[44] A. Gibbard, Manipulation of Voting Schemes: A general result, Econometrica 41 (1973) $587-602$.

[45] M. A. Satterthwaite, Strategy-proofness and Arrow's conditions: Existence and correspondence theorems for voting procedures and social welfare functions, Journal of Economic Theory 10 (1975) 187-217.

[46] R. Dong, Combinatorial Clock Auction for Airport Time Slots: An Agent-Based Analysis, Tech. Rep., Harvard University, senior thesis, 2005. 